Revue des patrimoines

$41 \mid 2019$

Patrimoines gastronomiques. Définitions, typologies et enjeux de conservation

\title{
Cerisiers, pêchers et ananas : des cultures de pointe dans les potagers royaux en Île-de-France dans la seconde moitié du XVIII ${ }^{\mathrm{e}}$ siècle
}

Cherries, peaches and pineapples; fruit growing in the royal vegetable gardens in the Île-de-France region during the second half of the eighteenth century

\section{Marion Rouet}

\section{(2)enEdition}

Journals

Édition électronique

URL : http://journals.openedition.org/insitu/25466

DOI : 10.4000/insitu.25466

ISSN : 1630-7305

Éditeur

Ministère de la Culture

\section{Référence électronique}

Marion Rouet, « Cerisiers, pêchers et ananas : des cultures de pointe dans les potagers royaux en îlede-France dans la seconde moitié du xvıII siècle », In Situ [En ligne], 41 | 2019, mis en ligne le 09 décembre 2019, consulté le 19 décembre 2019. URL : http://journals.openedition.org/insitu/25466 ; DOI : 10.4000/insitu.25466

Ce document a été généré automatiquement le 19 décembre 2019.

\section{(c) (i) $\odot$}

In Situ Revues des patrimoines est mis à disposition selon les termes de la licence Creative Commons Attribution - Pas d'Utilisation Commerciale - Pas de Modification 4.0 International. 


\section{Cerisiers, pêchers et ananas : des cultures de pointe dans les potagers royaux en île-de-France dans la seconde moitié du XVIII ${ }^{\mathrm{e}}$ siècle}

Cherries, peaches and pineapples; fruit growing in the royal vegetable gardens in the Île-de-France region during the second half of the eighteenth century

\section{Marion Rouet}

1 À la lecture de l'appel à contribution pour ce numéro d'In Situ consacré au patrimoine gastronomique, nous avons retenu certains éléments de la définition du «repas gastronomique » donnée par l'Unesco à travers le filtre de nos recherches en histoire des jardins : cet «art du» bien manger [...] met l'accent sur [...] le plaisir du goût, l'harmonie entre l'être humain et les productions de la nature. Parmi ses composantes importantes figurent [...] l'achat de bons produits, de préférence locaux, dont les saveurs s'accordent bien ensemble [... $]^{1}$.» Notre société actuelle associe la recherche du goût aux conditions de culture et d'exploitation des sols, ce qui implique un savoir-faire particulier. C'est donc par le biais du jardin que nous aborderons la question du goût pour les cerises, les pêches et les ananas dans la seconde moitié du XVIII ${ }^{e}$ siècle.

Dans un premier temps, nous avions songé à la culture des primeurs au XviII siècle. Très luxueuse, cette dernière était décriée tant au niveau du goût que du fait qu'elle soit « contre-nature ». Poursuivant notre travail de réflexion autour des productions qui impacteraient le paysage, et dans le cas présent le jardin, ainsi qu'autour des gestes techniques, nous avons déplacé notre attention sur la folle course technologique qui a eu lieu au cours du xviII ${ }^{\mathrm{e}}$ siècle autour du développement des serres pour des arbres fruitiers du fait de la «nouveauté ». Cela prend en compte l'idée de la précocité mais aussi celui de l'acclimatation des cultures fruitières afin de garnir la table du roi.

Pour parvenir à ce résultat, Louis XV va mettre en place, dès la seconde moitié du XVIII siècle, de nouvelles infrastructures dans certains jardins royaux. Le domaine de Choisy 
constitue l'un des exemples les plus frappants en Île-de-France et connaît alors de nombreuses transformations. Le roi modifie constamment l'environnement du château et multiplie la création de jardins. Pour son amusement, il fait faire de petits jardins particuliers, réservés à son usage, dans lesquels il encourage l'innovation technologique et pratique lui-même le jardinage. Une des passions du roi se matérialise à travers le développement des serres dans les jardins de Choisy, pour lequel Louis XV prend plaisir à faire abattre les arbres qui sont jugés nécessaires dès 1748 . Outre ce nouveau visage donné aux jardins royaux, la production précoce de pêches et d'ananas nécessite de nouvelles technologies ainsi qu'un personnel qualifié.

\section{Le développement du domaine de Choisy (Choisy-le- Roi, Val-de-Marne)}

Le domaine de Choisy est révélateur de tous les aménagements faits par le roi dans la seconde moitié du XVIII ainsi que de l'état d'esprit qui régnait alors dans les jardins royaux autour de la production de cerises, pêches et ananas. Le site se trouvait en bord de Seine et proche de la forêt de Sénart, ce qui séduisit Louis XV (fig. 1). Il acheta pour 100000 écus le château et le domaine, qui figuraient dans la succession de la princesse de Conti, décédée en mai 1739. La seigneurie de Choisy-sur-Seine devient celle de Choisy-le-Roi.

Figure 1

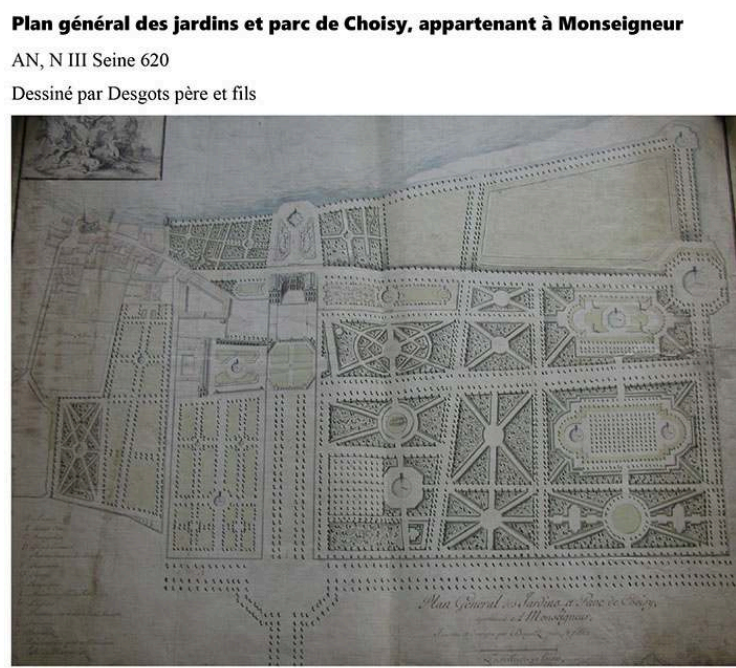

$\begin{array}{ll}\text { Légende: } & \\ \text { A. Avenues } & \text { I. Maison de monsieur Rolingue } \\ \text { B. Avant-cour } & \text { L. Eglise } \\ \text { C. Orangerie } & \text { M. Pavillon sur le bord de la Seine } \\ \text { D. Grande terrase } & \text { N. Cerisaie } \\ \text { E. Parterre devant le château } & \text { O. Pommiers } \\ \text { F. Réservoir } & \text { P. Pépinières quatre mendiants } \\ \text { G. Potager } & \text { Q. Salle des maronniers } \\ \text { H. Basse-cour } & \end{array}$

Plan général des jardins et parc de Choisy, appartenant à Monseigneur. AN, N III Seine 620. Dessiné par Desgots père et fils, xvıl siècle.

Phot. Marion Rouet. (C) Marion Rouet. 
5 À partir de 1752, le roi entreprend de grands travaux sur l'ensemble du domaine, qui acquiert le statut de demeure royale. Rivale de Marly, voire de Compiègne ou Fontainebleau, Louis XV n'y jouit plus de l'intimité et de la tranquillité premières. Sur l'emplacement du potager du domaine, il fait donc construire de 1754 à 1756 un « petit château » complété, en 1757, par un nouveau jardin appelé le jardin particulier du roi. La place accordée au jardin potager au sein de ces nouvelles réalisations nous interroge sur l'importance que revêtent les productions qui en sont issues.

\section{Le grand potager}

6 Lorsque le roi acquiert le domaine de Choisy, il contient bien évidemment un potager. Il est prévu qu'une avenue de quatre rangs de cerisiers de toutes variétés confondues serve d'entrée principale au "grand potager ». Situé le long de l'avant-cour, ce premier potager de Choisy, lors de la période étudiée, était composé de carrés. Un nouveau potager est mentionné en 1743 (fig. 2). Cependant, la destruction de l'ancien potager n'est ordonnée qu'à partir du mois d'avril 1750 afin de réaliser le jardin particulier du roi. En effet, le jardin commence à s'aplanir et la distribution doit être tracée. Les terres d'une partie de l'ancien potager doivent alors être déposées dans une rue se situant entre les deux potagers pour la relever à la même hauteur que le nouveau potager. Par ce moyen, un espalier doit être planté le long du mur du potager neuf lorsque le vieux mur sera détruit. En 1749, le grand carré du nouveau potager est entièrement remblayé, les plates-bandes le long des murs de clôture sont défoncées. Il reste alors une partie du carré où doivent être plantés les arbres en quinconce à remblayer. La plantation des arbres le long des murs du grand carré est prévue pour le mois de novembre. Il semble que ce potager soit construit en terrasse. Au mois d'avril 1750, le nouveau potager commence à prendre forme : 13 carrés sont fumés et plusieurs d'entre eux sont plantés et semés. 
Figure 2

Plan des châteaux et des jardins de Choisy-le-roi

(gravé par Le Rouge, 1783)

BN, Cabinet des estampes, Hd-89a-Pet. fol.

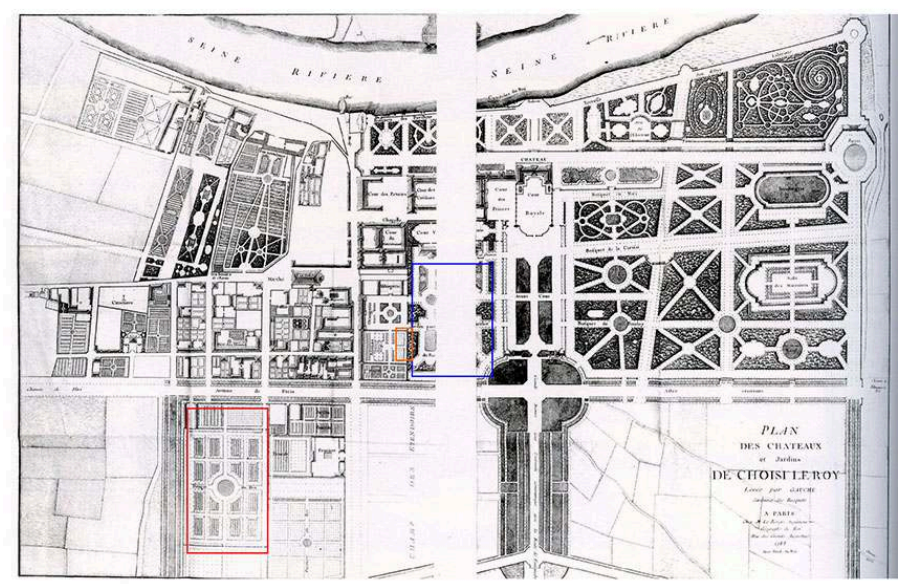

Légende:

Ancien potager du roi, devenu le jardin particulier du roi

Nouveau potager du roi de Choisy (grand potager)

Petit potager ou potager particulier du roi.

Georges-Louis Le Rouge, "Plan des châteaux et des jardins de Choisy-le-Roi », Jardins anglo-chinois à la mode ou Détail des nouveaux jardins à la mode, Paris, 1783. BnF, Cabinet des estampes, Hd-89aPet.fol.

(C) BnF.

7 Le nouveau potager devient alors le « grand potager » afin de le différencier du « petit potager » ou du " potager particulier du roi ». Il contient en 1766 près de 15 arpents de cultures, ce qui lui confère alors aux yeux de l'administration royale un caractère de grandeur assez exceptionnel. Il est composé de plusieurs parties ${ }^{2}$ (fig. 3) : tout d'abord, un grand enclos (8 arpents 50 ) divisé en 16 carrés de légumes, qui sont bordés d'arbres fruitiers. Il appartient donc à la famille «du jardin dit coupé, autrement dit compartimenté $»^{3}$. Sa structure est similaire au Potager du roi à Versailles puisque son centre est occupé par un grand bassin. Ensuite, pour répondre au goût de Louis XV pour les cerises, il y a une cerisaie de 2,5 arpents ${ }^{4}$, dans laquelle se trouvent également d'autres arbres fruitiers de plein vent. Ce potager comporte également un enclos pour les gros légumes, une figuerie ainsi qu'un jardin pour les couches de melons et les primeurs ( $1 / 2$ arpent cultivé toute l'année). 
Figure 3

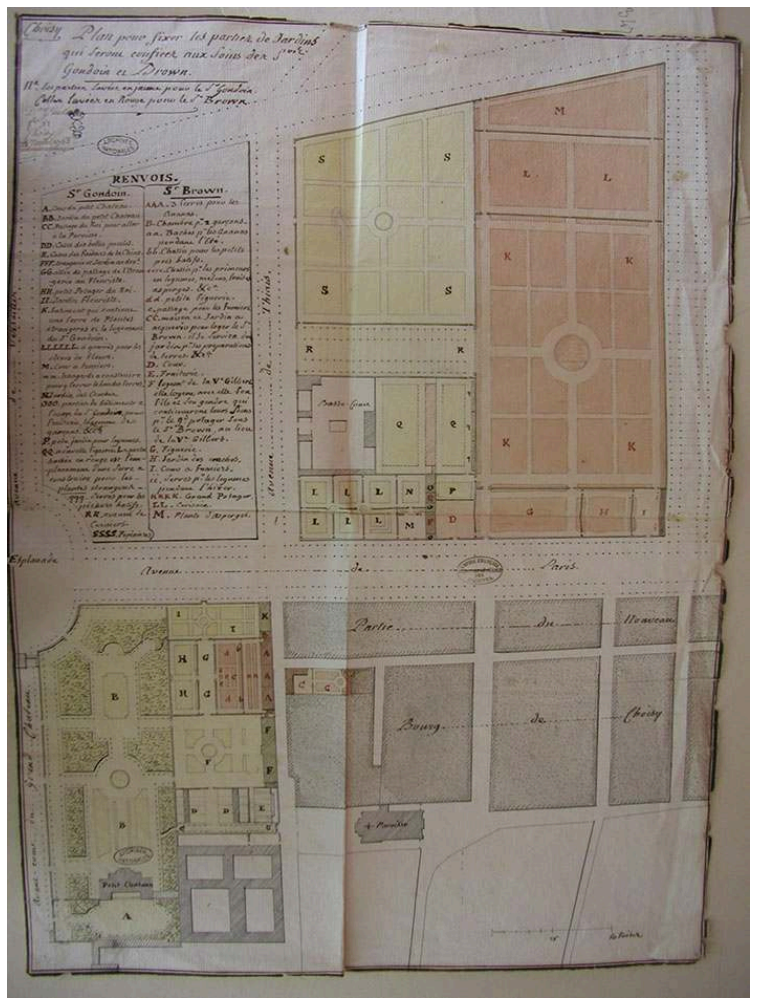

Plan pour fixer les parties de jardins [de Choisy] qui seront confiées aux soins des sieurs Gondoin et Brown, 1766 ?. AN, O' 1347-227.

Phot. Marion Rouet. (c) Marion Rouet.

\section{Le potager particulier du roi}

Les travaux de maçonnerie du potager particulier du roi ont débuté en octobre 1754. Dès cette date, le contrôleur des Bâtiments du département de Choisy s'est associé au jardinier fleuriste afin de choisir dans des pépinières de Vitry ${ }^{5}$ les arbres fruitiers destinés à ce jardin, en prenant en compte l'exposition. Des arbres fruitiers palissés en espaliers en agrémentent tous les murs. En 1758, il est demandé pour le potager particulier du roi 5 cerisiers de Hollande et 5 cerisiers griotte en haute-tige. Cette liste comporte des arbres demi-tige (10 pruniers Reine-Claude, 4 poiriers Beurré et 4 pêchers Royale) ainsi que des arbres nains (10 pruniers Reine-Claude, 5 pêchers Grosse Mignonne, 4 cerisiers de Hollande et 3 poiriers Saint-Germain). D'autre part, 100 pieds de vigne de différentes variétés y figurent. Nous pouvons donc observer la variété des espèces présentes dans ce jardin (à l'exception des pommes), qui trahit le goût immodéré de Louis XV pour les cerises. Ce jardin comporte des carrés et des plates-bandes, qui doivent fournir des légumes toute l'année. 
Figure 4

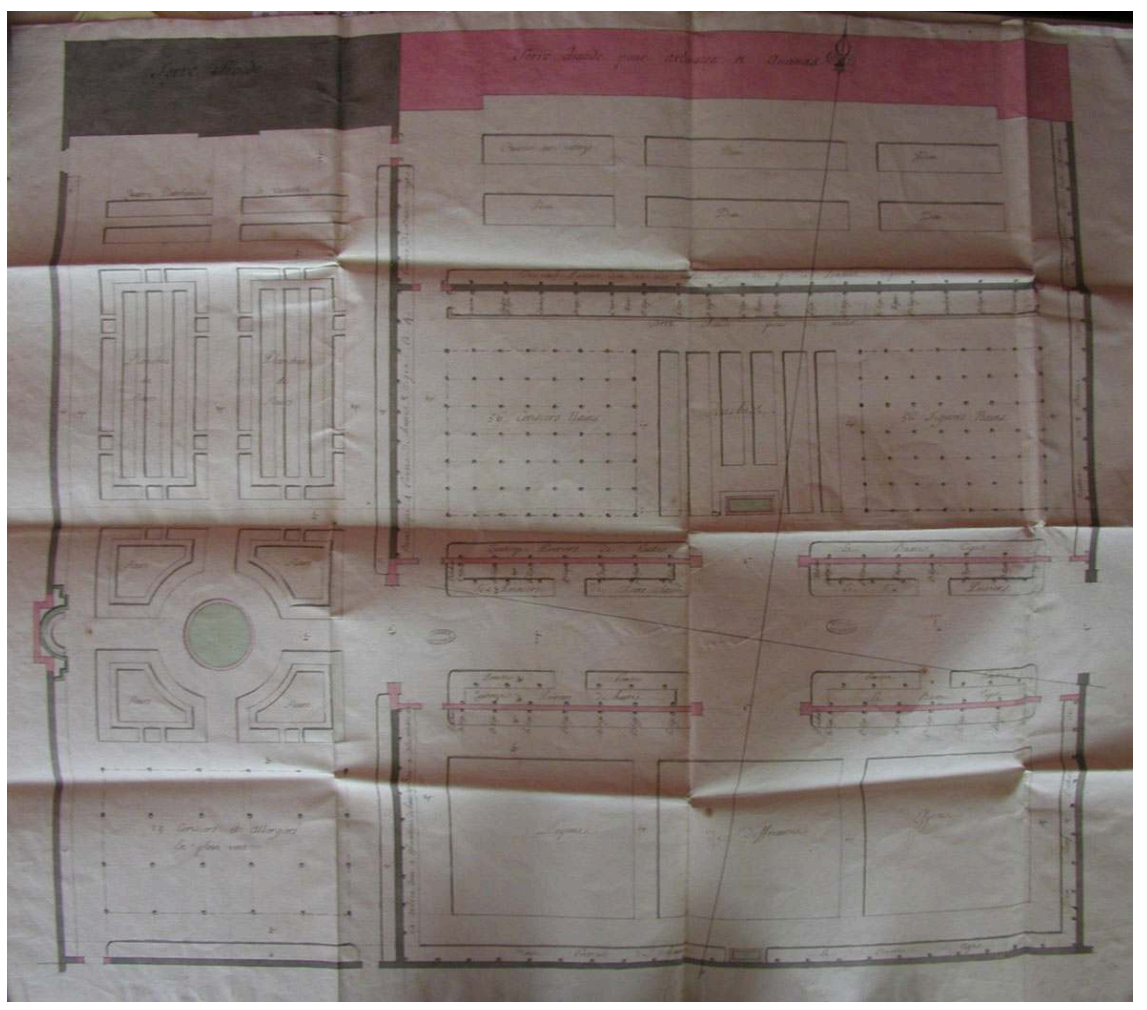

Le potager particulier du roi de Choisy et son environnement (1755). AN, O11351/36.

Phot. Marion Rouet. (c) Marion Rouet.

9 Nous possédons un projet de 1755 portant la mention " pour faire et parfaire ${ }^{6}$ (fig. 4) concernant l'environnement du potager particulier du roi. D'après ce plan, le jardin particulier du roi était composé de quatre ensembles: le jardin fleuriste, le potager particulier du roi, une petite figuerie et un espace dédié aux serres. Le premier comportait quatre planches de jacinthes, deux grandes planches de fleurs, un bassin entouré de fleurs ainsi qu'une petite cerisaie de 24 cerisiers et albergiers (variété d'abricotier) de plein-vent. Le potager du roi se composait de trois carrés espacés par des allées de 6 pieds de large ${ }^{7}$, où devaient être cultivées différentes espèces de légumes. En symétrie, la petite figuerie comptait un carré de 56 cerisiers nains, un carré de couches avec un point d'eau et un carré de 56 figuiers nains. D'autre part, le jardin particulier inclut deux petites serres: une serre froide pour les plantes étrangères et une serre chaude pour les arbustes et ananas. À cela s'ajoutent 6 caisses avec vitrage (fig. 5) et une serre chaude pour les fruits. Cette dernière désigne un espalier sur lequel doivent reposer des châssis vitrés. 


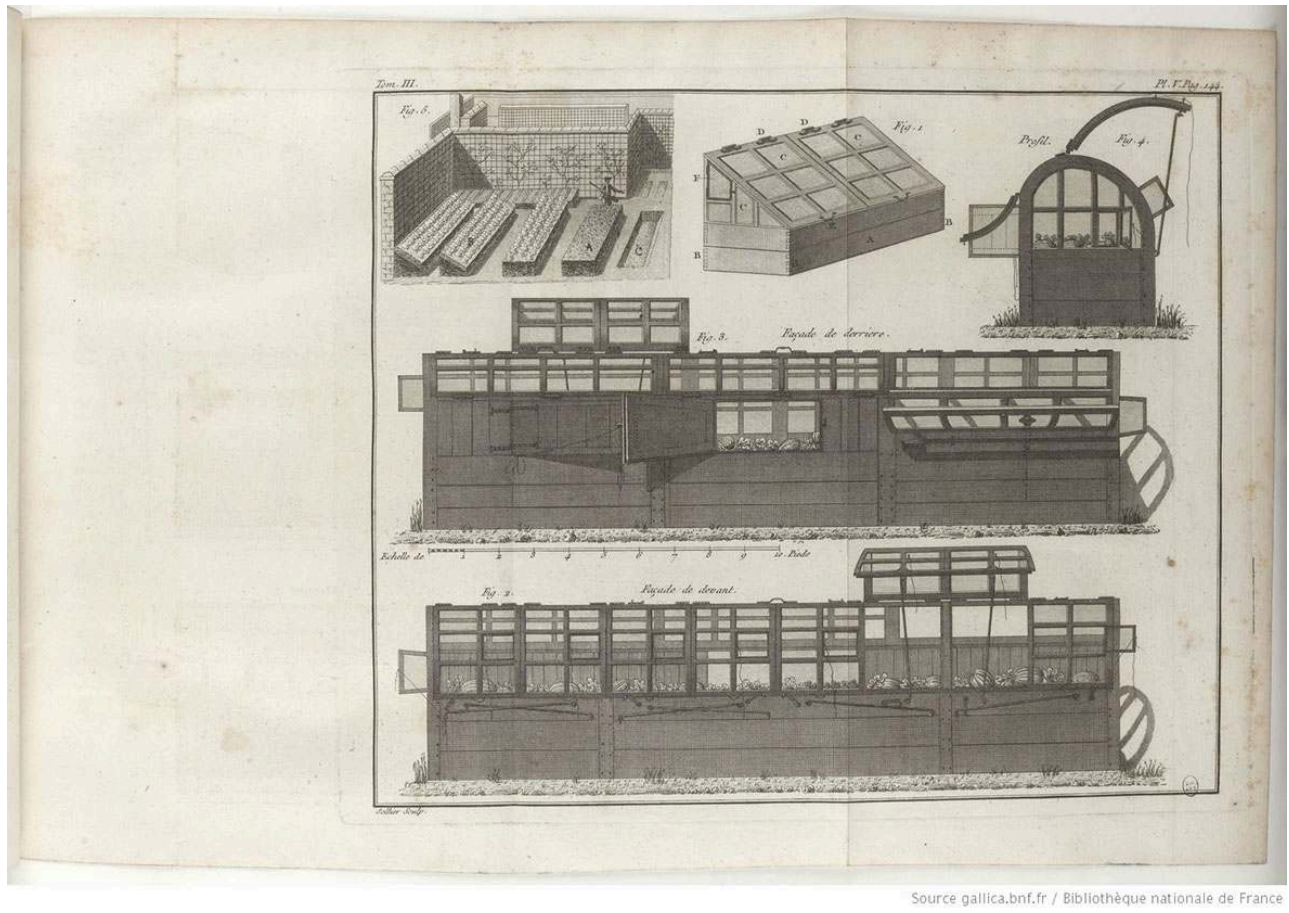

ROZIER, François. Cours complet d'agriculture. Paris : Hôtel Serpente, 1784, t. III, pl. V, insérée entre les p. 144 et 145 .

(C) BnF.

10 Compte tenu de la précision du plan de 1755 , nous connaissons la manière dont les arbres fruitiers étaient agencés dans ces jardins. Les plates-bandes, larges de 4 pieds ${ }^{8}$, devaient contenir à l'ouest 9 abricotiers de haute-tige, 9 abricotiers de basse-tige, 4 poiriers de haute-tige et 4 poiriers de basse-tige. À l'est sont figurés 19 pêchers de haute et basse-tige ainsi que 7 poiriers Bon Chrétien. Enfin, sur les murs de séparation des différents jardins orientés au nord ou au sud, sont dessinés 19 poiriers, la serre chaude pour les fruits, 14 poiriers. Dans l'allée permettant de joindre le jardin fleuriste à l'orangerie, une nouvelle structure se dessine à l'aide de deux plates-bandes de 4 pieds : des pêchers en espaliers en alternant haute-tige et basse-tige et sur le devant (peut-être en buisson) 6 pruniers Reine-Claude et 6 poiriers. En bordure du potager particulier du roi, nous retrouvons la même idée mais illustrée par d'autres espèces : des poiriers en espaliers et des pommiers sur le devant. À l'intérieur du potager particulier du roi se trouvent 14 pêchers et 20 poiriers palissés.

Le potager particulier du roi est pourvu des dernières innovations techniques tout au long de la seconde moitié du XVIII ${ }^{\mathrm{e}}$ siècle. Louis XV ordonne en janvier 1759 de planter des figuiers en pleine terre sous les châssis de son petit potager. Par la suite, le roi fait faire à Ange-Jacques Gabriel le plan d'un petit verger en juillet 1761 qu'il veut créer à côté de sa terrasse pour y planter des cerisiers. Il doit délivrer sur le champ la somme nécessaire soit 4000 livres, sinon rien ne pourra être fait. Enfin, une nouvelle serre est implantée dans le petit potager du roi en 1773 pour les primeurs de fleurs. Son usage semble s'intensifier puisque le jardinier fleuriste est censé, d'après son marché d'entretien de 1776, y cultiver des ananas, des figuiers en caisses, des primeurs, des fleurs et des arbustes. 
12 Le domaine de Choisy comporte également des jardins spécialisés dans un type de culture. Le bâtiment de l'orangerie, construit entre 1752 et 1753, est divisé en deux serres. Le jardin des ananas est dédié à ces derniers mais il abrite également des serres à arbustes et plantes étrangères ainsi que des serres plus modestes pour les primeurs (petits pois). D'autre part, sont recensés en 1773 des châssis pour "avoir de bonne heure des melons, concombres et autres legumes $»^{9}$ et des quinconces de figuiers.

\section{Le goût pour les fruits rouges}

13 «Les cerises étant les premiers fruits que nous offre le retour de l'été, leur goût étant très agréable, leur suc raffraichissant et surtout très séduisant par le piquant d'aigrelet ou d'acide qui se rencontre dans la plus grande partie, il n'est pas étonnant que ces fruits soient généralement aimés et recherchés. ${ }^{10}$

14 Afin d'avoir des cerises dans la nouveauté, c'est-à-dire de façon précoce, vers le mois de mai, Jean-Baptiste de La Quintinie, responsable du jardin potager du roi à Versailles, les cultivait en espaliers dans la mesure du possible. Lorsque les fruits étaient trop aigres, avaient peu de chair, un gros noyau ou la peau épaisse, le jardinier du roi recommandait de les servir en compote. Le potager de Versailles offre la plus grande diversité avec 21 variétés commandées au cours du XVIII siècle. Outre la célèbre cerise de Montmorency, la Hollande et l'Angleterre sont les noms des variétés de cerisiers sélectionnées dans le but d'avoir des arbres qui produisent de façon hâtive. Mme de Case, épouse d'un fermier général, cherche d'ailleurs à obtenir «des greffes des beaux cerisiers » de Choisy en 1786, il semble même que son fils ait obtenu la promesse d'obtenir « les cerisiers même $»^{11}$.

15 Louis XV ne se satisfait plus des résultats obtenus grâce aux espaliers et aux jardins spécifiques. Il décide de mettre en place de nouvelles infrastructures afin d'obtenir des cerises de façon plus hâtive dans l'année. Outre la cerisaie du grand potager et la création du jardin particulier du roi, il ordonne pour Choisy la création d'une serre à cerisiers, attestée en février 1768. Une nouvelle serre est mentionnée en janvier 1769 dans l'allée aux cerisiers. De plus, Louis XV accorde un bon du roi le 22 juillet 1769 pour la construction de serres pour les cerisiers. La multiplication des serres à cerisiers trouve son explication dans le fait que ces arbres se fatiguent assez rapidement. Le parti pris est donc de démolir les serres pour les reconstruire dans la cerisaie à un autre endroit pour forcer d'autres sujets. En effet, les cerisiers ne peuvent pas rapporter deux années de suite lorsqu'ils sont sous serre car ils sont trop épuisés. Le contrôleur du département de Choisy parvient à la conclusion que ce système est peu rentable :

16 «Ce qu'il y a de certain [...], c'est que cette maniere de cultiver les cerisiers rapporte bien peu de fruits pour la depense qu'elle occasionne; cependant comme ces serres ont paru plaire au roi, je vous supplie de lui demander si son intention est que l'on en reconstruise deux cette année comme on a fait les années precedentes $[. . .]^{12}$.»

17 Le jardinier fleuriste, Jacques Gondoin, souhaite également la disparition de ces serres car elles monopolisent un garçon jardinier supplémentaire. De ce fait, Louis XV renonce à poursuivre la culture des cerisiers sous serre pour cette année-là.

18 Après les expériences menées en 1768 et 1769 par le jardinier fleuriste sur les cerisiers, Louis XV renouvelle son désir de poursuivre et de perfectionner les serres à cerisiers lors du voyage à Choisy en novembre 1771. Le roi ordonne alors le début des travaux 
pour de nouvelles serres en laissant 25 louis au concierge du château. Cependant, le jardinier affirme qu'il faut au plus tard commencer à chauffer la serre à Noël, ce qui paraît impossible du fait des délais de construction et des conditions climatiques. Louis XV repousse son projet à l'année suivante.

Afin d'assouvir sa passion, Louis XV va ramasser lui-même les fruits sur les arbres à l'aide d'échelles à marchepied, qui sont dûment contrôlées en mai 1771. Pour la somme de 740 livres, 5 grandes échelles doubles à marchepied sont réalisées par un entrepreneur en menuiserie pour le roi en 1773. Dans les Confessions ${ }^{13}$, Jean-Jacques Rousseau relate un souvenir ému de la cueillette des cerises, symbole d'une vie champêtre heureuse et quelque peu insouciante (fig. 6).

Figure 6

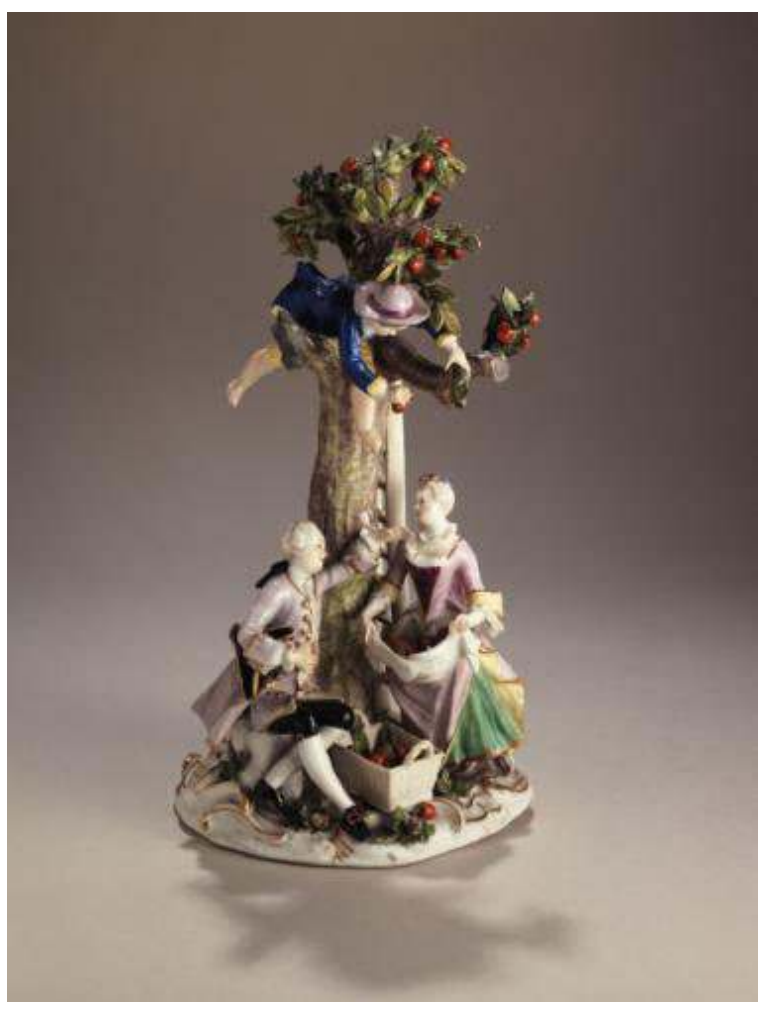

La Cueillette des cerises. Johann-Joachim KAENDLER, porcelaine, xviiie s. Paris, Musée du Louvre (J 880).

Phot. Thierry Ollivier. @ Musée du Louvre, Dist. RMN-Grand Palais.

Comme son prédécesseur, Louis XVI semble très friand de ce fruit, qui se fait très rare en 1780 : «le peu qu'il y en a [de cerises] devient encore plus precieux et demande à etre conservé avec tout le soin possible pour le service du Roy qui paroit avoir pour ce fruit plus de gout que pour beaucoup d'autres ${ }^{14}$.» Des mesures sont ainsi prises à Choisy afin de permettre aux cerises de parvenir à maturité. Les jardiniers ont commencé par faire garder les cerisiers par des hommes qui tapaient sur des poêles et des chaudrons ou bien qui utilisaient des sonnettes. Ces moyens sont peu efficaces car les oiseaux s'habituent au bruit. Aussi, l'inspecteur des potagers royaux demande-t-il au directeur des Bâtiments du roi d'autoriser les jardiniers à tirer eux-mêmes sur les oiseaux. L'administration ordonne une chasse aux pinsons, merles, grives, loriots et sansonnets durant la saison de maturité des cerises. L'administration des Bâtiments du 
roi consulte par la suite Louis XVI sur le sort des jardins fruitiers et potagers de Choisy "que Sa Majesté paroit s'en occuper sous le double rapport de l'utilité de son service et de son amusement $»^{15}$. Sous couvert de légèreté, le goût pour les cerises est donc plus complexe qu'il n'y paraît car il requiert une domestication de la nature. Le plaisir ressenti lors de la dégustation contraste avec les lourdes et les coûteuses infrastructures nécessaires, qu'elles soient financières, humaines ou naturelles.

Bien que n'ayant pas le statut d'arbres fruitiers et ne bénéficiant pas des nouveaux procédés technologiques, les arbustes produisant des fruits rouges ont été appréciés des plus grands, notamment de Louis XV. Si les cerises et les fraises sont particulièrement appréciées, il ne faut pas oublier les groseilles et les framboises. Les mois de juin et de juillet sont propices au sein des domaines royaux aux envois de cerises, bigarreaux, groseilles, fraises et framboises. Ainsi, quatre corbeilles de cerises de Choisy et quatre corbeilles de fraises de Meudon sont expédiées en express en juin 1750, auxquelles sont joints 18 artichauts violets de Choisy et une botte de jacinthes, pour être présentés au roi le lendemain matin. À cette occasion, l'administration des Bâtiments du roi préconise de grouper les envois avec ceux du jardinier du potager de Versailles, qui se font le vendredi. En juillet 1776, Jacques-Louis Le Normand, jardinier du Potager du roi de Versailles ${ }^{16}$, informe le directeur général des Bâtiments que c'est le temps des groseilles et que s'il désire faire de la gelée, la saison des framboises va bientôt s'achever. Cela implique donc que ces deux fruits rouges sont mélangés pour réaliser de la gelée.

\section{Les pêches}

22 Florent Quellier affirme que la pêche détrône la poire au cours du XVIII ${ }^{e}$ siècle du fait du développement de la culture des pêchers en espalier «dans les clos aristocratiques et bourgeois, mais aussi, pour répondre à la demande parisienne, dans des clos paysans comme au village de Montreuil, célèbre pour sa manière de cultiver les pêches. $\aleph^{17}$ À l'image des cerisiers, les potagers royaux usent pour la culture de la pêche d'artifices autres que l'étude de l'exposition et la conduite des arbres en espalier ${ }^{18}$.

La serre chaude, donc chauffée, bouleverse les conditions de culture. Dès le mois de février 1755 , le contrôleur du département de Choisy constate que dans la serre chaude où se trouve le poêle, les pêches sont déjà nouées (passées de l'état de fleur à celui de fruit) tandis que dans l'autre partie de la serre, les arbres sont en retard: ils sont sur le point de fleurir. Les serres chaudes nécessitent une grande quantité de terreau à mélanger avec de la terre : cela est valable pour les pêchers mais aussi les figuiers, les ananas et les fraisiers. En août 1755, le jardinier fleuriste demande 100 tombereaux de terreau, soit 1100 livres de dépense puisque chaque voiture est facturée 11 livres.

Cependant, les arbres fruitiers supportent difficilement l'intensité de la vie sous serre: beaucoup de pertes sont dénombrées, notamment parmi les pêchers. En septembre 1757, le contrôleur du département de Choisy veille à ce que les châssis soient transportés sur de nouveaux espaliers afin de laisser reposer ceux qui ont rapporté et de façon à ce que le roi puisse bénéficier de pêches en primeur l'année suivante. Une autre solution au problème est donnée dans le marché d'entretien du jardinier fleuriste en 1776 : ce dernier réchauffe tous les ans un tiers de l'étendue de la serre. 

Les châssis sont régulièrement refaits à neuf puisque les arbres, après avoir fructifié, sont mis au repos pendant quelques années. De plus, suivant la disposition des terrains, il est parfois impossible de réutiliser les anciens châssis. Le jardinier fleuriste de Choisy se plaint en 1757 des derniers, réalisés en sapin, qui réclament de nombreuses réparations sous peine de voir la pluie s'infiltrer. Durant l'été 1760 , le roi demande la construction de fourneaux et de châssis tout au long de l'espalier de pêchers de Choisy, qui se situe dans un enclos entre les petits jardins où sont élevées les fleurs et l'avenue des cerisiers qui conduit au grand potager. Il espère ainsi obtenir des pêches de bonne heure l'année d'après. En septembre 1768, une nouvelle serre à pêchers est en construction sur le domaine.

Dans le domaine royal de Bellevue, des serres à pêches sont achevées en janvier 1765. Il reste à effectuer la plantation. Pour pallier la disparition précoce des pêchers sous serre, le contrôleur du département prévoit dès le $1^{\text {er }}$ mars 1765 d'envoyer le jardinier choisir 3 douzaines d'arbres dans les pépinières royales, tant en demi-tige qu'en nain, parmi les variétés les plus convenables à cet usage. Ces arbres seront élevés en dehors du potager dans une bonne exposition de façon à remplacer les arbres défaillants sous serre. Lors du regarni ${ }^{19}$ annuel en octobre 1765 , ce sont 36 pêchers qui sont réclamés par le contrôleur pour pallier l'épuisement de certains arbres. Sont choisies quatre variétés tant en tige qu'en nain: 6 Grosse Mignonne, 6 Madeleine rouge, 3 Chevreuse (fig. 7) et 3 Violette (fig. 8), la préférée du roi.

Figure 7

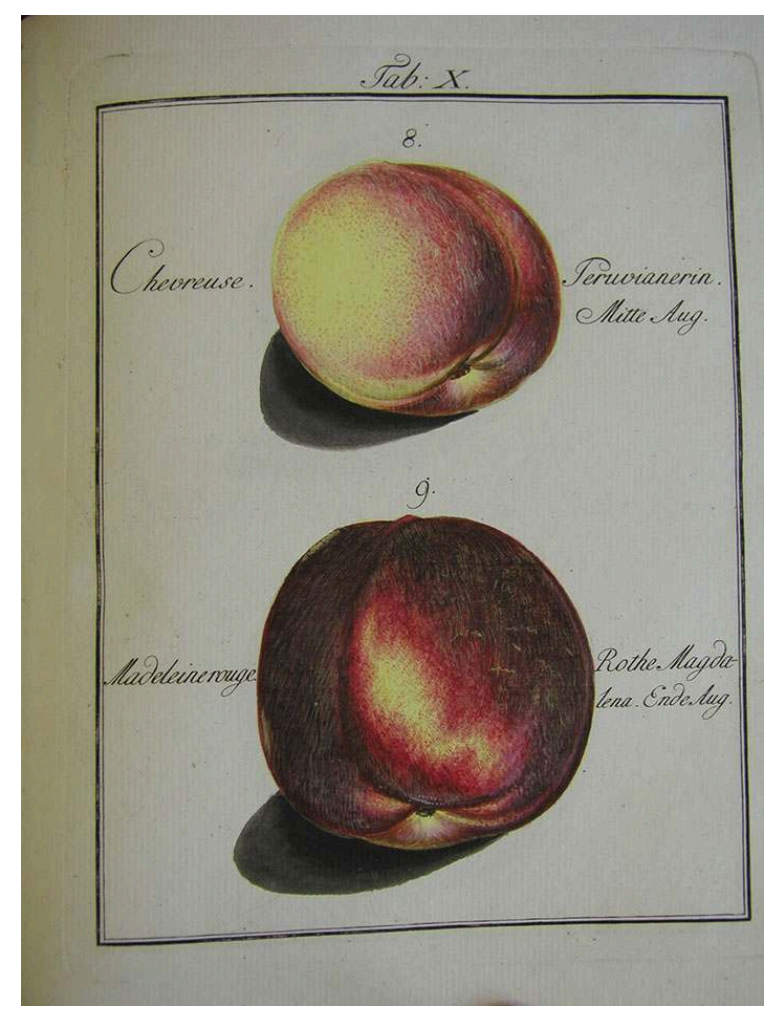

MAYER, Johann. Pomona Franconica. Nuremberg : chez Adam Wolfgang Winterschmidt, 1779, t. II, tab. $X$, Chevreuse et Madeleine rouge.

Phot. Marion Rouet. (C) Marion Rouet. 
Figure 8

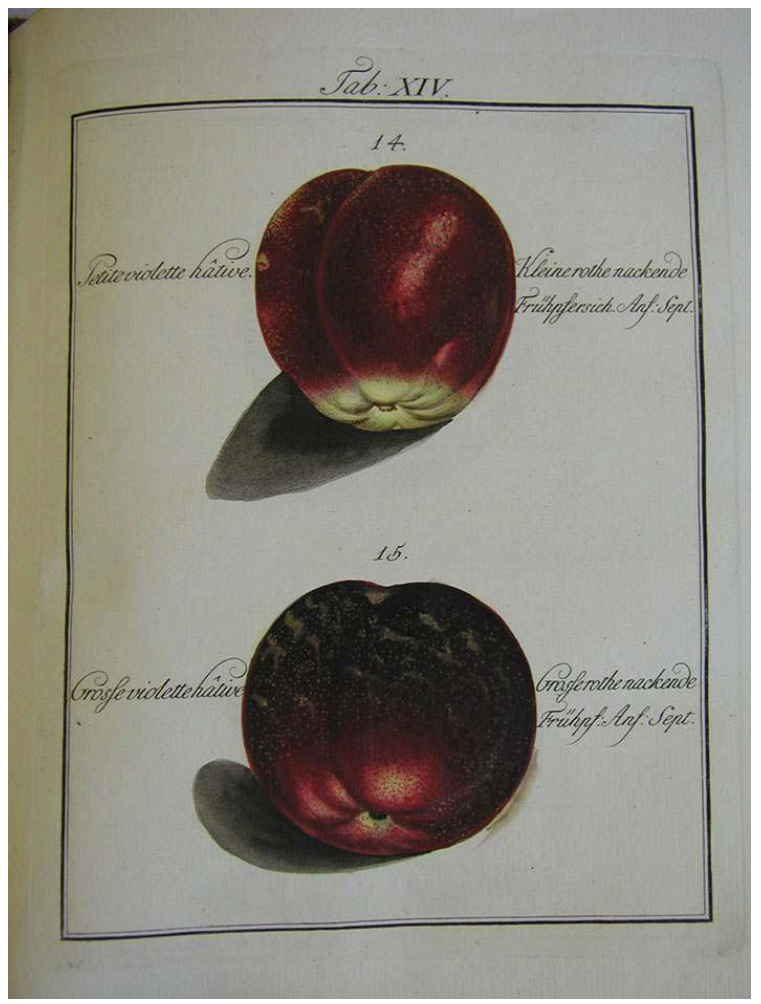

MAYER, Johann. Pomona Franconica. Nuremberg : chez Adam Wolfgang Winterschmidt, 1779, t. II, tab. XIV, La pêche violette hâtive.

Phot. Marion Rouet. (c) Marion Rouet.

Cependant, la culture des pêchers sous serre se trouve à l'opposé de l'idéal d'insouciance liée à la cerise. Du fait de la présence de fourneaux, les serres chaudes entraînent des accidents. C'est le cas à Choisy en mars 1763, où le feu dévaste une partie des serres à pêchers :

Le feu a pris par le fourneau à une des trois serres des pêchers. Le garçon jardinier qui est toujours de veille s'en est apperçu sur le champ. Il a jetté 3 ou 4 arrosoirs qu'il a trouvées pleines d'eau. Ce n'auroit été rien sans le vent affreux qu'il faisoit qui a fait gagner le feu bien vite. Il a été reveiller le sieur Gondoin fils qui est venu aussitôt avec un autre garçon et en moins d'une demi-heure ils ont tout eteint. Le danger étoit trop pressant pour qu'un d'eux put se détacher pour aller éveiller d'autre monde. Il n'y a que 9 pieds de longueur de chassis qu'il faudra refaire, mais la fumée a gaté tout ce qu'il y avoit de fruit dans cette serre. Heureusement encore dans ce malheur que les premieres pêches qui sont dans la serre à côté ne sont aucunement endommagés, non plus que celles qui devoient succeder à celles qui sont perdues. Le sieur Gondoin fils assure que cela n'empechera pas ces arbres de rapporter l'année prochaine ${ }^{20}$.

De ce fait, l'utilisation de serres chaudes implique l'emploi d'un personnel qualifié :

"Vous scavez l'attention que le Roy donne à ses jardins fleuristes et potager. Les serres et les couches ont si fort changé depuis peu de tems la methode et l'art de la culture des fleurs, des fruits, des plantes orangeres [...] qu'il est difficile de trouver des gens qui y soient exercées ${ }^{21}$.»

L'abbé Schabol ${ }^{22}$, expert en arboriculture fruitière, est ainsi chargé d'examiner les arbres fruitiers du domaine de Choisy en 1762. Cependant, cela suscite chez les 
jardiniers chargés de l'entretien une certaine animosité. En effet, le spécialiste est appelé lorsque survient un problème et il met bien souvent en cause le travail mené jusqu'alors par le jardinier.

J'ay écrit, Monsieur, à M. l'abbé Roger [Schabol], personnage tres expérimenté pour les maladies des arbres et surtout des arbres fruitiers. L'intention de Sa Majesté e[s]t qu'il examine tous ceux des jardins de Gondoin et surtout ceux des serres chaudes. Vous le laisserez agir et opérer dans tous les secours qui luy seront necessaires. Comme il a reussy dans une infinité de jardins par sa méthode, sur le succès qu'il fera, je pourray bien le faire parcourir les autres maisons royales : il n'y aura pas de mal pendant qu'il travaillera dans ces petits jardins-la de luy faire voir le grand potager qui aura, je crois, besoin de son secours. Dittes à Gondoin que mon intention est qu'il se prête à tout ce que demandera M. l'abbé Roger, et vous me rendrez compte tres exactement de tout ce qui se passera la-dessus, et des depenses que l'on exigera de vous.[...]

P.S. Surtout, que Gondoin n'aille pas faire le jaloux et chercher en dessous à nuire aux opérations de M. l'abbé Roger ${ }^{23}$.

31 Dès le mois de décembre, l'abbé Schabol a commencé à palisser et à tailler différents pêchers des jardins de Choisy. Il rédige un mémoire afin de remédier et de corriger les défauts qu'il a pu observer sur la gestion des arbres fruitiers. Il note que ces arbres ont été plantés de façon trop rapprochée: de ce fait, il faut leur apporter beaucoup d'engrais sans l'enfouir complètement. L'abbé accorde une grande importance à ce que l'on apporte du fumier aux arbres fruitiers. De plus, il fait lever des arbres dans les pépinières de Vitry : en effet, un des points les plus essentiels de sa méthode consiste à lever les arbres avec leurs racines et non de les arracher.

L'affrontement entre l'abbé Schabol et le jardinier fleuriste du domaine de Choisy est assez brutal dans la mesure où l'abbé a adopté une technique particulière, à savoir celle des maraîchers de Montreuil. C'est la taille et le palissage à la loque qui constituent le secret (fig. 9, fig. 10). 
Figure 9

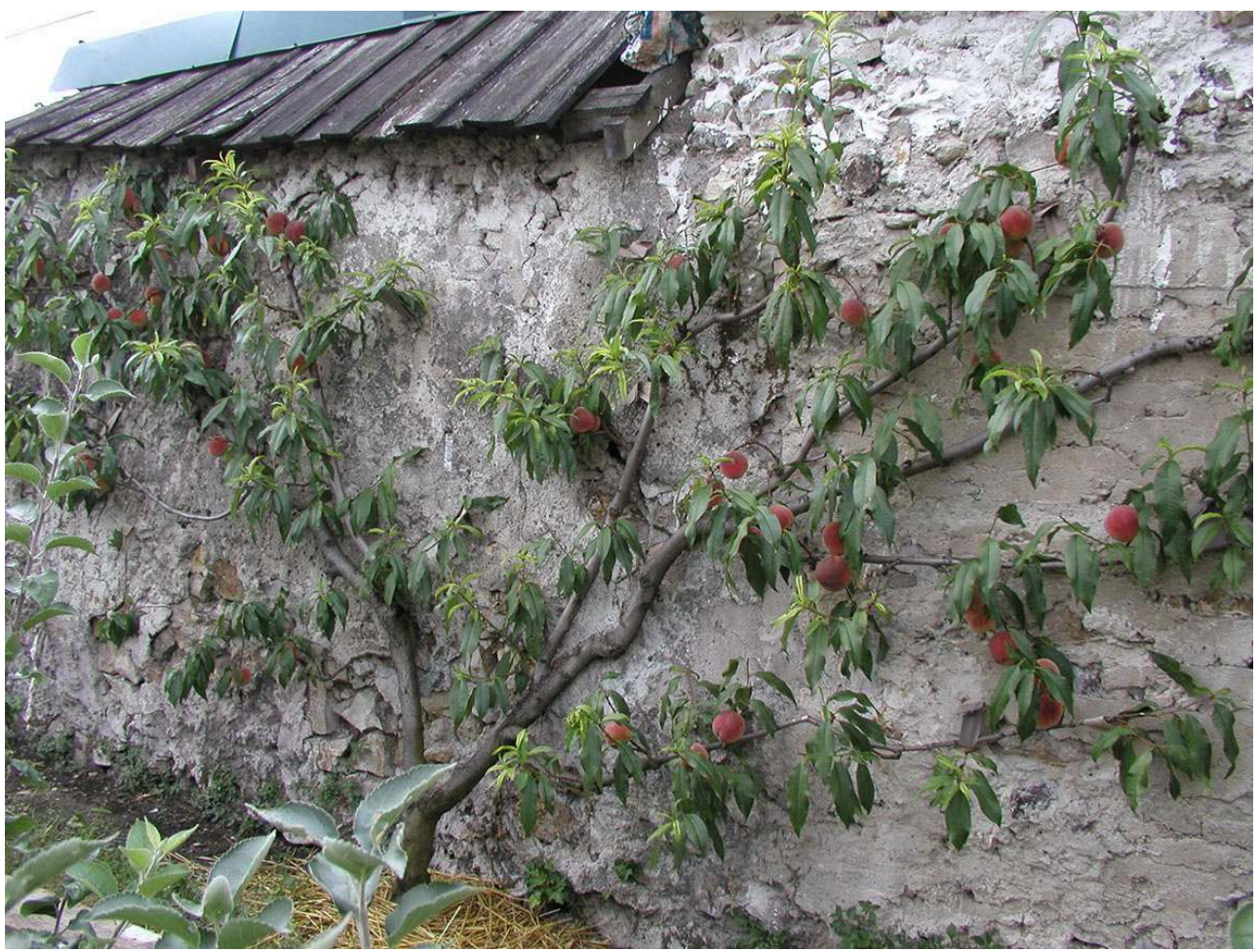

Pêcher taillé «à la manière de Montreuil », Association des murs à pêches de Montreuil.

Phot. Marion Rouet. (c) Marion Rouet.

\section{Figure 10}

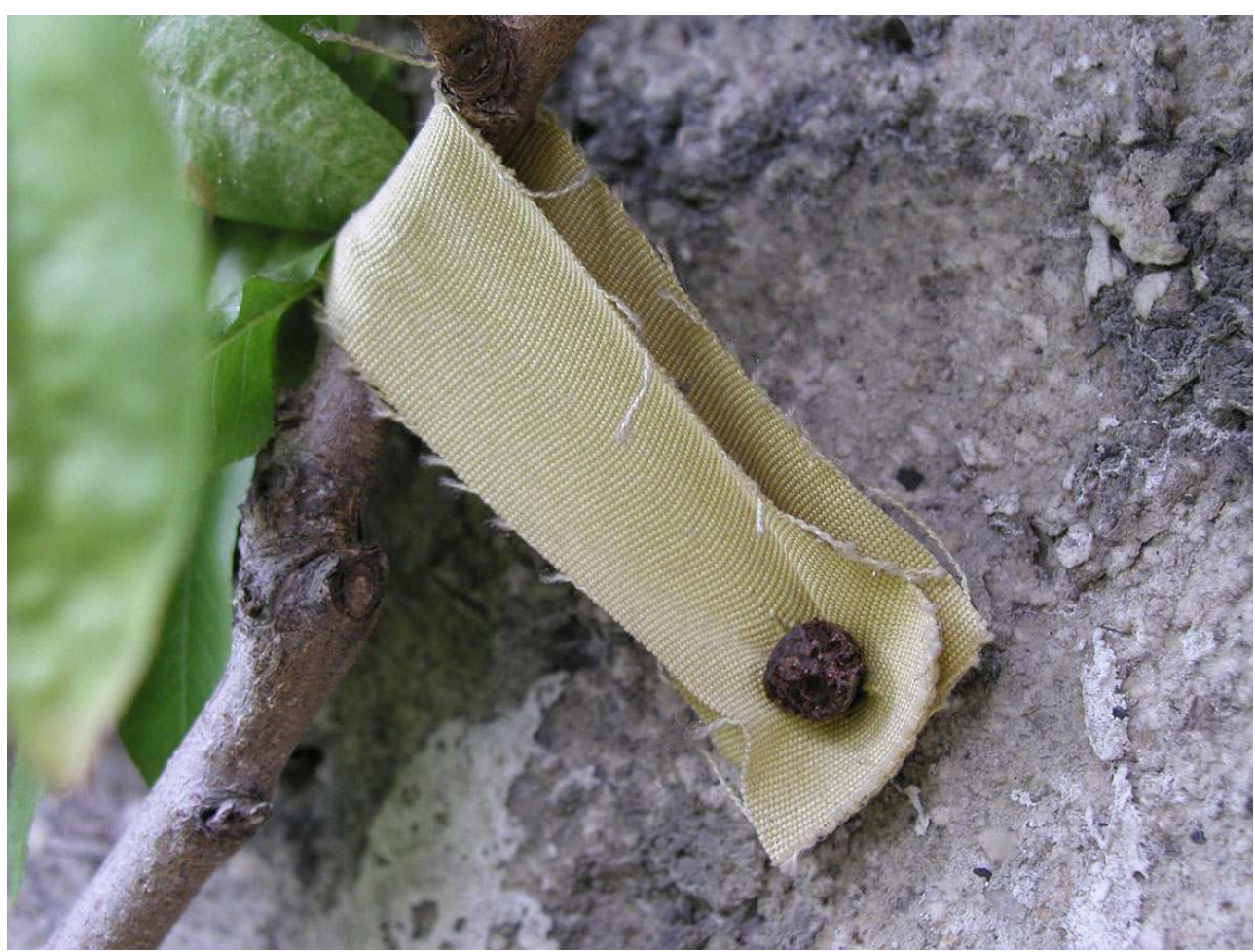

Palissage à la loque, Association des murs à pêches de Montreuil. Phot. Marion Rouet. (c) Marion Rouet. 
Pour cela, il suffit de supprimer le canal direct de la sève et de donner à l'arbre la forme d'un $\mathrm{V}$ en supprimant les branches verticales des espaliers. Pour compléter l'ensemble, il convient de palisser l'arbre de manière oblique à l'aide de clous et de bandes de tissu découpées sur des murs de plâtre. Le jardinier fleuriste de Choisy s'oppose à l'abbé Schabol sur la question de la taille. Pour lui, l'abbé ne rabat pas assez les arbres et leur laisse trop de bois, ce qui constitue un des principaux reproches fait à la méthode employée à Montreuil. Aujourd'hui encore, l'Association des murs à pêches de Montreuil (MAP) perpétue la tradition de ce savoir-faire ${ }^{24}$. Elle cherche à préserver un paysage spécifique tout en assurant la transmission de gestes et de techniques de production. Paradoxalement, c'est ce qui distingue l'action de l'abbé Schabol au sein des potagers royaux. En effet, les arbres fruitiers conduits en espaliers dans ces jardins sont palissés sur des treillages de bois afin de garantir à l'ensemble une esthétique. La production n'est pas le seul critère qui entre en compte dans l'entretien d'un potager royal.

Schabol est rappelé durant l'année 1764 : au mois d'octobre, les 21 rôles de dépenses de l'abbé depuis novembre 1762 s'élèvent à plus de 19000 livres. "Le roi se promenant aujourd'huy l'a vu à l'ouvrage et ne lui a rien dit. Sa Majesté a parcouru tout le grand potager et le sieur Brown a accompagné ${ }^{25}$. La disgrâce de Schabol est annoncée car Louis XV a tout mis en œuvre pour employer Alexandre Brown à Choisy. Bien que l'abbé soit présent tout le mois de juillet 1764 , les productions promises ne sont pas là et il n'est pas improbable que Brown et Gondoin aient œuvré en sa défaveur. En août, 5065 pêches sont dénombrées pour 236 pêchers, «ce n'est pas autant qu'il avoit fait esperer, mais beaucoup plus que le S[ieu]r Gondoin n'en attendoit. Plus 366 poiriers 455 poires, c'est bien peu $»^{26}$. De plus, l'abbé Schabol a perdu son seul soutien, le directeur des Bâtiments du roi, en tenant des propos diffamatoires à son encontre en présence du roi. Aussi, le marquis de Marigny doit justifier auprès du roi, le 28 octobre 1764, l'état de sa consommation personnelle des fruits de Choisy. Je suplie très humblement Votre Majesté de me permettre une représentation.

Plusieurs temoins m'assurent qu'il a été dit par le S. abbé Roger en presence de Votre Majesté qu'il n'étoit pas etonnant que l'on ne portât point de pesches du jardin de Choisy au Roy, attendu qu'il en avoit vu porter deux cens chés moy et que l'on me donnoit tout le fruit de Choisy et qu'il etoit bien singulier qu'on n'en portât point chés le Roy.

Je donne ma parolle d'honneur à Votre Majesté que lorsque le S. abbé Roger a osé tenir un pareil propos, il n'avoit été porté chés moy dans toute l'année, du jardin de Gondoin, que 15 pesches et deux melons ; que recemment, il en a encore été aporté chés moy une douzaine et que c'est tout. [...]

J'ose prendre la liberté de suplier très humblement Votre Majesté de m'authoriser à donner des ordres pour qu'on ne laisse plus entrer dans ses jardins de Choisy le S. abbé Roger, seul moyen de parvenir à faire retablir les arbres et les figuiers dont le mauvais état ne peut que confirmer qu'il n'a ny theorie ni pratique certaines qui puissent determiner à luy en confier le soin ${ }^{27}$.

À cette occasion, le roi décide donc d'évincer l'abbé Schabol de ses jardins et de négocier un arrangement autour de l'installation du sieur Brown. Dès lors, les jardiniers Brown et Gondoin ont pour ordre d'interdire l'accès aux jardins à l'abbé Schabol tandis que les jardiniers du parc ne doivent en aucun cas suivre les conseils qu'il aurait pu leur prodiguer. Ainsi, l'abbé Schabol va se heurter aux pratiques des jardiniers royaux déjà en place ainsi qu'au jardinier anglais Alexandre Brown, auquel Louis XV fait appel pour réussir la culture des ananas à Choisy à partir de 1763. 
L'esthétique du jardin et l'acclimatation d'une nouvelle culture au sein du potager ont eu raison du savoir-faire de Montreuil.

Le goût n'est jamais mentionné dans cette guerre technique, c'est à l'esthétique des productions que l'on fait référence (fig. 11). Cependant, lors de l'arrivée de l'abbé Schabol à Choisy, celui-ci constate dans l'avenue des cerisiers que l'espalier de pêchers est à fumer car il est « affamé » par les asperges cultivées au pied et par la présence des cerisiers. Le jardinier de Choisy répond que l'espalier a été fumé l'année précédente et n'en a donc pas besoin avant trois ans. L'utilisation du fumier est pourtant au cœur des débats sur le goût dans les traités de jardinage jusqu'à la fin du XviII siècle. Dans son Traité de la culture des pêchers, de Combles pense que fumer les pêchers donne non pas plus de goût mais plus d'eau et de délicatesse aux fruits car ils sont mieux nourris ${ }^{28}$. Certains auteurs insistent sur le fait que le fumier doit être bien consommé, et non pas frais, avant de planter les arbres en espalier de façon à ce qu'il ne communique pas son mauvais goût aux fruits.

Figure 11

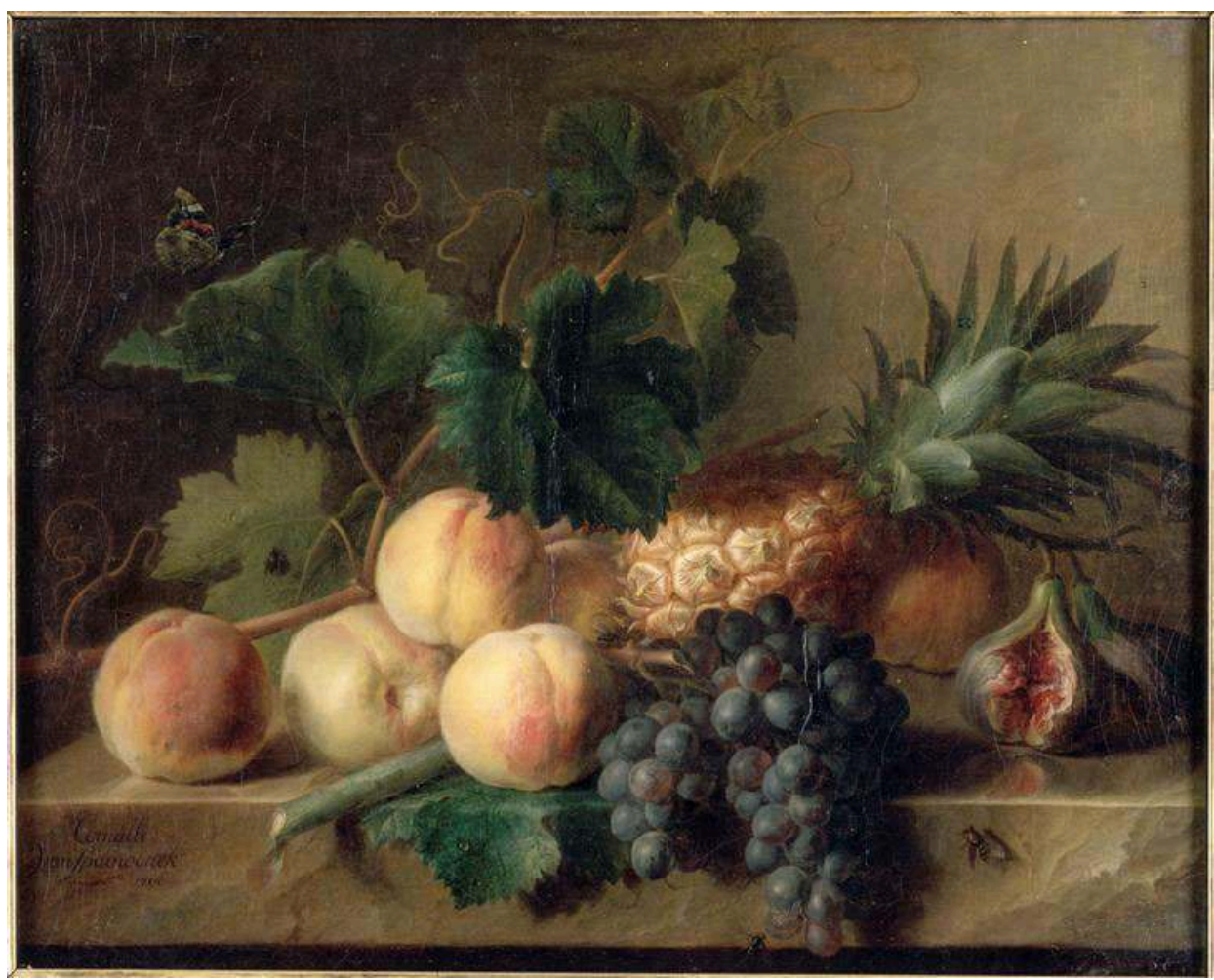

Nature morte aux pêches et à l'ananas. Cornelis Van SPAENDONCK, 1798, huile sur toile, 43 x $54 \mathrm{~cm}$. Paris, musée du Louvre, département des Peintures (RF 2853).

Phot. Jean-Gilles Berizzi. @ RMN-Grand Palais (musée du Louvre).

\section{Les ananas}

Dans la seconde moitié du XVIII ${ }^{\mathrm{e}}$ siècle, la culture de l'ananas est beaucoup plus dynamique que celle des figuiers : elle fait figure de culture de pointe dans certains potagers royaux. Louis-Sébastien Mercier mentionne dans le Tableau de Paris $^{29}$ que les jardins parisiens de M. Boutin ${ }^{30}$ et des ducs de Chartres et de Biron ${ }^{31}$ sont parmi les plus 
remarquables (fig. 12). En outre, il conseille aux amateurs d'aller à Navarre près d'Évreux chez le duc de Bouillon afin d'admirer les procédés du jardinier anglais qui y élève plus de 4000 pots d'ananas.

Figure 12

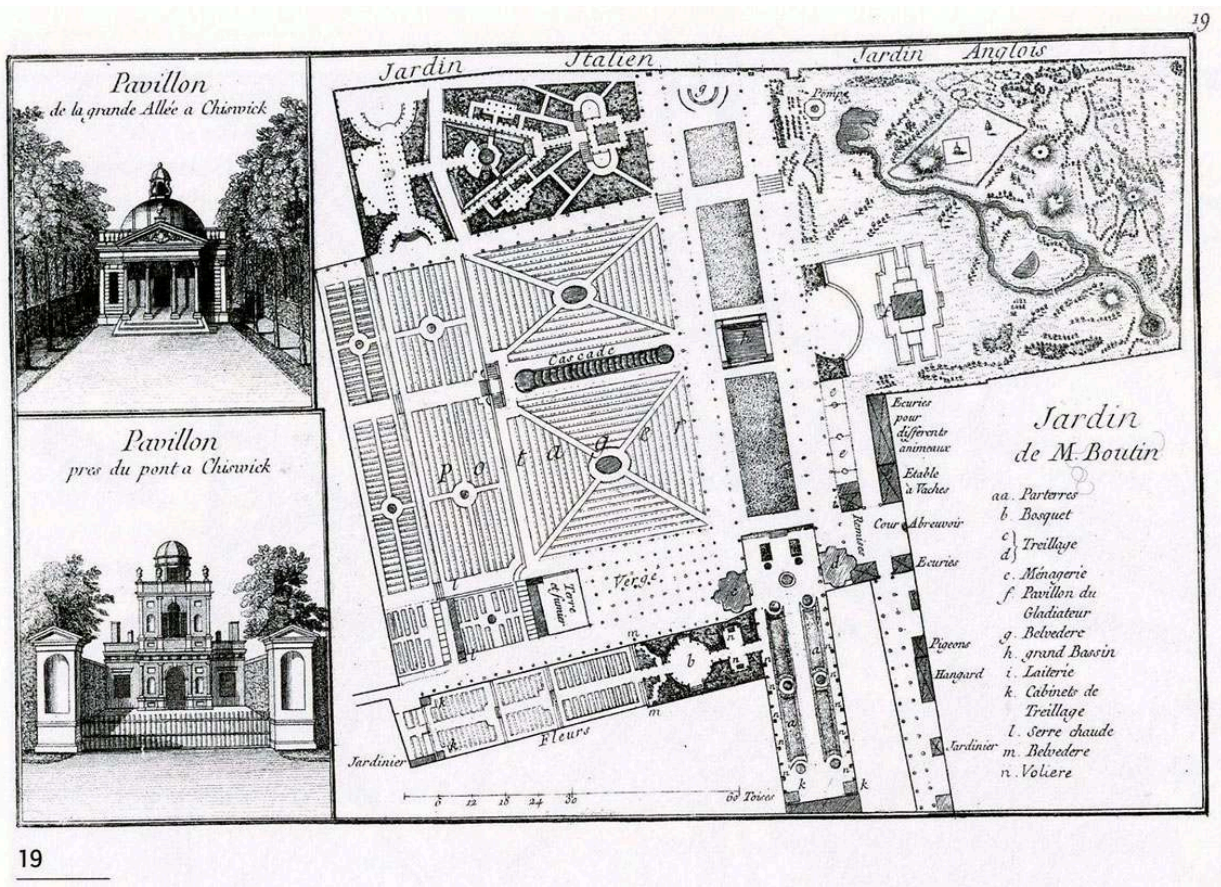

Georges-Louis Le Rouge, «Plan du jardin de M. Boutin », Jardins anglo-chinois à la mode ou Détail des nouveaux jardins à la mode, Paris, 1775. BnF, Cabinet des estampes, Hd-88-4.

(C) BnF.

La culture de l'ananas réclame un matériau spécifique, la tannée ${ }^{32}$. D'après le projet de marché d'entretien du jardinier du potager de Choisy en 1766, il semble que la composition des terres pour les ananas soit identique à celle des primeurs. À cette intention, il est fourni au jardinier tans, poudrettes, feuilles sèches, terres des environs du pays ainsi que du sable. En 1773, 40 voitures de terre attendent au bois de Boulogne pour les ananas de Choisy. De plus, il est demandé au jardinier « d'élever les plantes d'ananas de maniere qu'il y ait de ces fruits dans la plus grande quantité possible, gros et de la meilleure qualité $\aleph^{33}$. Il doit également veiller à perpétuer de jeunes plants d'ananas en œilletons et en couronnes ainsi que de cultiver les plantes étrangères qui sont restées dans les serres à ananas.

Pour ce faire, Brown, le nouveau jardinier à la tête du potager de Choisy, est responsable d'allumer les fourneaux des serres et d'y entretenir le feu autant qu'il est nécessaire, à l'aide du bois qui lui est fourni et qu'il doit scier. Il semble que les jardiniers commencent à chauffer les serres à partir de la Toussaint. Outre la préparation des terres, le jardinier doit être vigilant quant à la propreté des serres en les balayant et en les nettoyant fréquemment. En septembre 1771, les marchands de bois refusent de livrer le domaine de Choisy du fait des trop grandes avances qu'ils ont consenties. Le contrôleur du département s'inquiète de la situation, les ananas et autres productions sous serre étant en péril. Il est vrai que l'ananas est une plante très fragile qui demande beaucoup d'attentions et de nombreuses installations. 

roi à Versailles (fig. 13) ${ }^{34}$. Le projet était à exécuter avant l'hiver. Large de 10 pieds $^{35}$, le système de chauffage de cette serre était compris dans le mur de soutènement. Les ananas recevaient directement la lumière par de petites vitres. Une facture de juin 1751 atteste de l'expédition depuis Amsterdam, par le biais de Bruxelles, d'une caisse de 50 couronnes d'ananas qui doivent donner des fruits en 1753 et d'une caisse de 50 plants d'ananas « qui doivent rapporter » (soit fructifier) en 1752. Ces derniers sont d'ailleurs beaucoup plus onéreux : un plant est facturé 50 sols la pièce tandis qu'une couronne revient à 20 sols pièce.

Figure 13

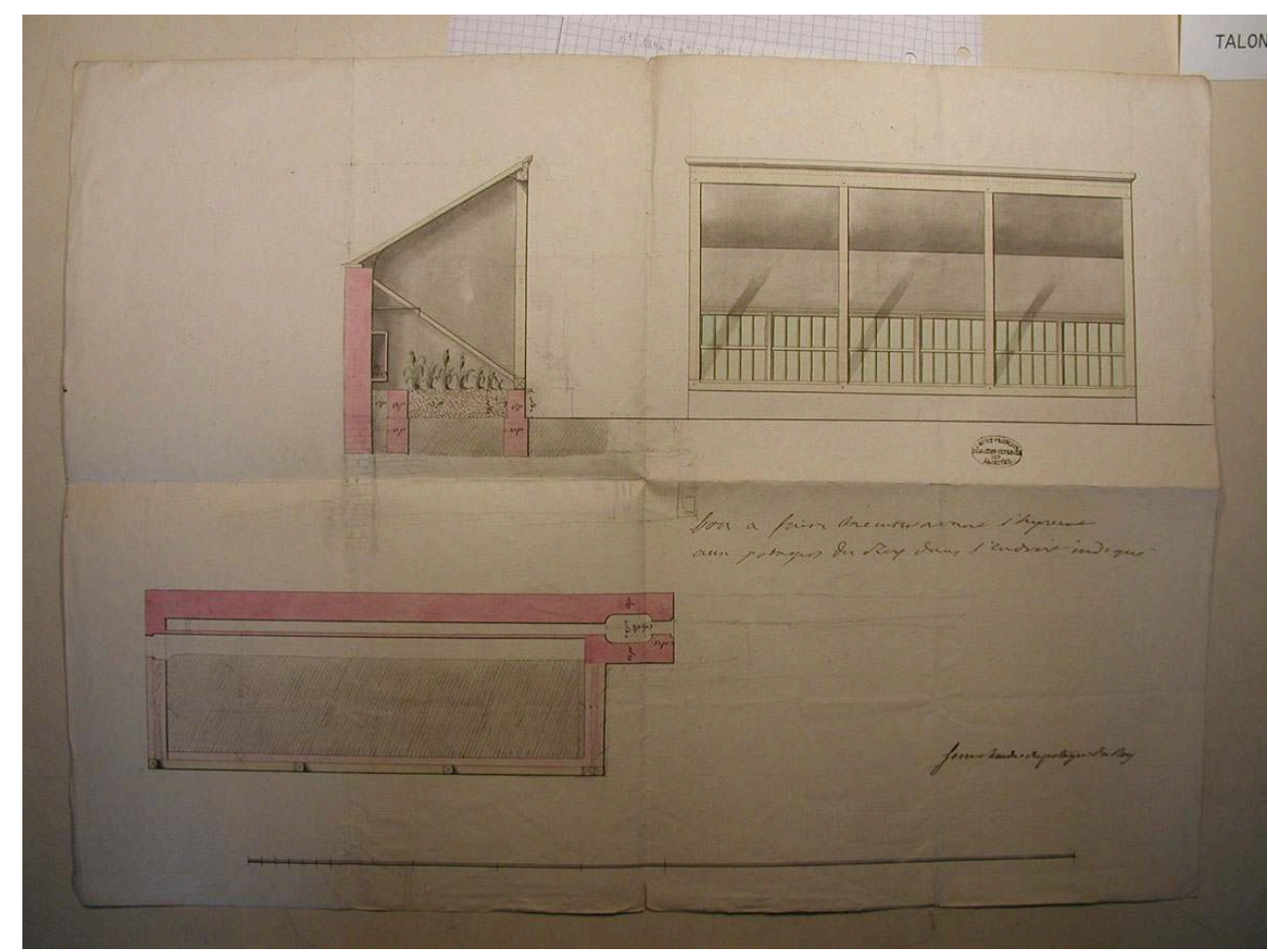

Plan, coupe et élévation d'une serre chaude (Versailles, entre 1737 et 1745). AN, $0^{1} 1846-5 n^{\circ} 22$.

Phot. Marion Rouet. (c) Marion Rouet.

Les premières serres à ananas construites sur le domaine de Choisy datent de 1754 . Pour rendre ces serres chaudes conformes à celles de Pâris de Montmartel, financier et propriétaire du domaine de Brunoy (Essonne), il s'avère nécessaire en 1755 de les pourvoir de stores de toile cirée verte suivant l'intention du roi. Pour la grande serre aux ananas et aux plantes curieuses de 25 toises de long, cela représente une dépense de 1760 livres pour 11 stores. Il en est de même pour les deux petites serres du potager particulier du roi. Les stores sont indispensables car ils permettent de recueillir lorsqu'ils sont baissés l'humidité ou la condensation déposée sur les vitres. Sinon, celleci retombe en gouttes sur les cultures, ce qui est jugé préjudiciable aux plantes. De plus, les stores interceptent une partie des vapeurs s'élevant vers le vitrage.

En 1756, la dépense concernant les ananas provenant de Hollande n'est pas rentable car sur les 100 pieds, seuls 12 sont sauvés par Gondoin, jardinier du domaine de Choisy. La responsabilité de ce dernier est mise en cause : considérant que ce sont des plantes 
exotiques, il les a placées dans une serre chauffée tandis que son fils, formé en Hollande, préconisait un endroit moins chaud. Cependant, Gondoin fils livre un témoignage intéressant depuis La Haye où il visite tous les jardins où se trouvent les plus beaux ananas. Il rapporte les prix qui ont cours là-bas : il faut compter 10 livres pour un pied sur bois, 6 livres pour les pieds les plus médiocres « mais qui sont encore belle plante $»^{36}$ et 8 livres pour les plus petits pieds. Afin de profiter de ce voyage, Louis XV a d'ailleurs approuvé de confier à Gondoin fils la mission d'acquérir des plantes et des fleurs pour les regarnis des jardins de Choisy et du potager de Versailles à concurrence de 10000 livres. La fleur la plus appréciée du roi étant la jacinthe, Gondoin fils remarque qu'il n'y a aucune comparaison possible entre la beauté des jacinthes de Hollande et celles de Choisy. Il se demande si cette différence n'est pas due à la qualité des oignons reçus en France. Nous pourrions étendre cette interrogation à d'autres cultures, y compris celle de l'ananas.

En mars 1757, 100 pieds d'ananas de trois ans quittent Amsterdam pour les regarnis des jardins du roi de Choisy, pour la somme de 1137 livres. De plus, le sieur Horneca ${ }^{37}$ envoie, malgré la rigueur de la saison, un superbe ananas afin qu'il soit présenté au roi s'il arrive en bon état. En 1758, une nouvelle commande de 400 pieds d'ananas est passée en Hollande, du fait de l'accroissement des serres chaudes de Choisy à la demande de Louis XV. Dans le courrier, il est stipulé que l'on veille à ce que les plants soient bien desséchés avant d'être emballés car l'année précédente, un grand nombre d'entre eux est arrivé pourri. Aussi, l'administration des Bâtiments du roi demande 50 plants d'ananas pour réparer le préjudice. Les regarnis se poursuivent à grand rythme puisqu'en mai 1759, ce sont 367 pieds d'ananas de deux ans (qui doivent produire en 1760) qui sont expédiés, pour un montant de 2518 livres.

De nouvelles serres chaudes sont construites à Choisy en 1761 après la destruction au mois de septembre de deux serres, dont l'une était en bois de chêne et l'autre en bois de sapin. Lors de l'arrivée du jardinier Brown en 1763, les serres sont divisées en trois parties qui forment trois serres de dimension égale et sur le même alignement. Les deux situées aux extrémités renferment la majeure partie des plantes étrangères tandis que celle du milieu sert aux ananas pendant l'hiver. Le jardinier anglais trouve cette serre insuffisante pour " mettre à l'aise pendant l'hiver la grande quantité d'ananas »" Il réclame que les trois serres soient affectées à la culture des ananas. Un projet de serre pour les plantes étrangères est donc étudié dans l'enclos des serres à pêches, suivant la volonté du roi. Ainsi, en 1776, le jardin des serres de Choisy contient une très grande serre vitrée, où les plants d'ananas et certaines autres plantes exotiques sont enfermés durant l'hiver, ainsi qu'une bâche couverte de châssis vitrés, où les ananas sont déplacés au printemps pour y passer l'été.

Du fait de l'arrivée du nouveau jardinier spécialisé et vraisemblablement de ses origines ainsi que du nombre de serres plus important, une nouvelle commande de plants d'ananas est passée en Angleterre par l'intermédiaire de Pierre Andrieux, marchand fleuriste et beau-père du botaniste Philippe-Victoire de Vilmorin ${ }^{39}$. Brown reçoit ainsi du sieur Paul Edé de Londres trois caisses contenant 240 plants d'ananas en mai 1764. Deux d'entre elles renferment des plantes en bon état tandis que la dernière recèle 40 grandes plantes et 15 à 20 petites pourries. Il est donc demandé un dédommagement au fournisseur par le biais du département de Choisy, ce qui est accordé. Nous possédons pour cette commande de plus amples précisions, à savoir la forme, l'âge, la variété et le prix du pied. Le jardinier a donc demandé 40 plants d'ananas à chair verte 
(greenfleshd pineapple), 35 plants pyramidal ou en pain de sucre des Barbades (sugard lof pine in Barbados), 20 plants pyramidal ou en pain de sucre à chair jaune (sugar lof pineapple with a yellow flesh), 15 plants couleur d'olive (olive pineapple). Les quatre premiers doivent porter des fruits en 1765 et sont facturés 6 livres 12 sols pièce. Les suivants sont moins âgés puisqu'ils doivent fructifier à partir de 1766 et représentent une dépense de 3 livres 12 sols par pied, ce qui représente un total de 1194 livres. La commande comprend donc également 50 jeunes plants d'ananas à chair verte, 50 jeunes plants pyramidal ou en pain de sucre des Barbades, 15 plants pyramidal ou en pain de sucre à chair jaune et 15 plants couleur d'olive. La commande est passée le 20 mars 1764. Les caisses sont réceptionnées à Calais le 13 mai et expédiées à Paris le 20 mai. Elles étaient accompagnées d'un ordre des fermiers généraux afin qu'elles ne soient pas ouvertes mais soumises à caution pour la douane de Paris.

D'autre part, ces commandes sont complétées par des achats chez des particuliers. Lors de son arrivée à Choisy, Brown a acheté pour près de 600 livres au maréchal de BelleIsle, secrétaire d'État à la Guerre et propriétaire du domaine de Bizy (Vernon, Eure) ${ }^{40}$, 150 pieds d'ananas dont 12 pieds portaient des fruits, 42 étant prêts à en porter et 96 pieds moins avancés. Ainsi dès le 2 août 1763, Brown récolte son premier ananas à Choisy et le fait porter au roi, qui séjourne à Compiègne ${ }^{41}$. De plus, il semble que des particuliers étrangers enrichissent les collections du roi. En effet, Mme Powell, de Londres, a fait passer un très grand panier de plants d'ananas à destination des jardins de Choisy en juillet 1764. De même, le financier Joseph Pâris-Duverney offre en octobre 1766 au roi des tulipes et des pieds d'ananas, que Brown doit aller choisir à Plaisance (Nogent-sur-Marne). En 1776, la provenance pour les commandes de pieds d'ananas semble plus diversifiée puisqu'il est fait référence aux îles, à l'Angleterre ou à la Hollande comme source d'approvisionnement possible en cas de besoin.

En outre, les serres sont remaniées et perfectionnées tout au long de la seconde moitié du XVIII ${ }^{e}$ siècle. Ainsi, un projet de serres ramené d'Angleterre par Nicolas Parant de Martigné, trésorier de France, est étudié en 1765 à Choisy. Le principe est de parvenir à « dégraisser » l'air des serres chaudes de la façon suivante :

Il consiste à établir à une des extremités plusieurs fourneaux ou poëles de brique, autour desquels on fait circuler l'air exterieur avant d'entrer dans la serre. À l'autre extremité, il pratique au plafond une ouverture d'environ 6 pouces de diametre jusqu'au dessus du comble, et dont l'embouchure tournante par le moyen d'une girouette est toujours opposée au vent. Il pretend que l'effet de cette ouverture est de pomper tout le mauvais air qui est toujours renouvellé par l'air nouveau qui vient des poëles ${ }^{42}$.

D'après lui, les Anglais et les Français sont encore dans le balbutiement du point de vue de la construction des serres. Aussi, il a imaginé une nouvelle serre qu'il appelle « un petit monde » car il fait « circuler l'air dans une chambre ou serre comme le soleil fait circuler l'air dans le monde ou comme le cœur fait circuler le sang dans les veines ", c'est-à-dire où l'air extérieur ne pénètre jamais. En effet, il pense que l'air se dégraisse en se raréfiant. L'expérience s'est limitée à l'exécution d'un modèle de petite taille.

L'année 1765 est très riche en projets pour les serres à ananas puisque le jardinier Brown en remet un qui est acceptét3 (fig. 14). Il proposait deux emplacements possibles, dans la melonnière, ou entre la pépinière et le jardin fleuriste. C'est ce dernier endroit qui est retenu par Louis XV en août 1765. Mais la construction d'une grande serre chaude nécessite d'empiéter sur la pépinière. De plus, le projet prévoit un 
logement pour le jardinier, une cour à fumier, la fruiterie du grand potager et un magasin pour stocker les stores et les matériaux nécessaires.

Figure 14

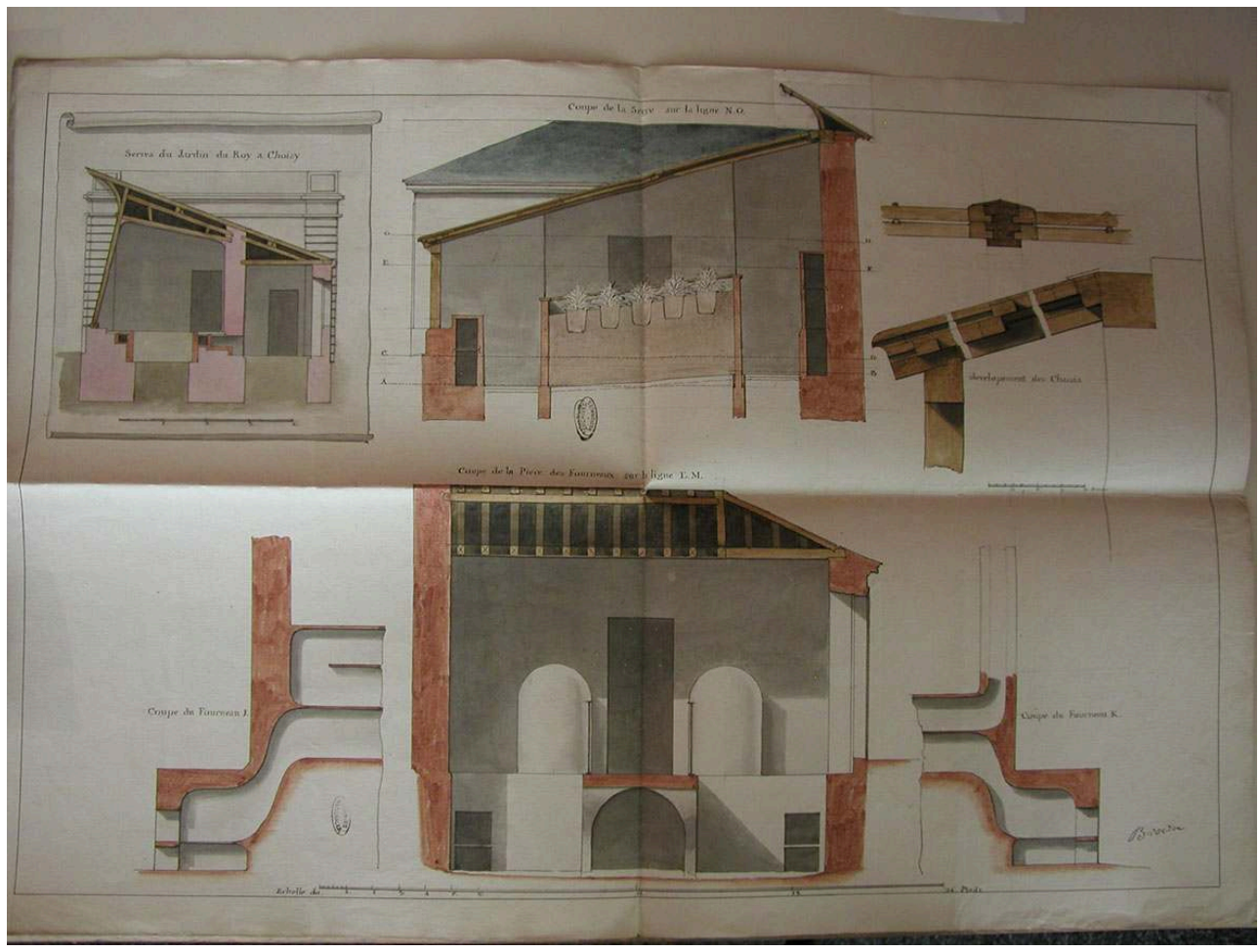

Projet d'une serre pour le jardin du roi de Choisy par Brown, jardinier du potager (1765). AN, O' 1347-513.

Phot. Marion Rouet. (c) Marion Rouet.

Brown joint un mémoire instructif à ses plans dans lequel il livre sa réflexion. Tout en énumérant les qualités de cette grande serre, il fait une critique de la serre existante dont il ne demande pas la destruction car elle peut servir d'ornement dans le jardin. De plus, elle peut accueillir les jeunes plants durant l'hiver. Visuellement, il cherche à en restreindre la taille tandis que son projet met en avant l'importance de la pièce à fourneaux et la simplification de la circulation. Par la présence des lettres alphabétiques, il cherche à démontrer le poids de sa recherche autour de la question de l'ensoleillement qu'il veut maximal, pour ne pas avoir à sortir les ananas de la serre. En effet, durant l'été, et ce dès le mois d'avril en 1763, les ananas du domaine de Choisy sont placés à l'extérieur des serres dans des bâches. Les serres sont chauffées à partir d'octobre.

Celle que l'on propose recevra les rayons du soleil toute la journée tant obliquement que perpendiculairement pandant tout le court de l'année, au moyen de ce que les plants d'ananas ne sortiront point de cette serre ils y produiront par l'effet du soleil leurs fruits avec succès au lieu que celle qui existe actuellement n'en reçoit les rayons qu'obliquement l'espace d'environ deux heures dans les jours les plus courts, et à mesure qu'ils croissent, le soleil s'elevan plus sur son horizon, les plants d'ananas qui se trouvent près du mur elevé n'en reçoivent que foiblement les rayons et dans les plus grands jours la moitié de cette même serre est privée du moindre effet de soleil, ce qui fait qu'on ne peut y laisser les ananas, lesquels sont si susceptibles que cet astre qui leur est très necessaire pendant l'eté pour produire leurs fruits avantageusement ${ }^{44}$. 
51 Ce projet comporte plusieurs fourneaux mais aussi de nouveaux châssis pour bénéficier d'un éclairage oblique et perpendiculaire, comme nous le voyons sur le plan en coupe ${ }^{45}$ (fig. 15). La serre, comprenant un pavillon à chaque extrémité, mesure par elle-même 10 toises. Un des pavillons doit abriter une chambre pour un garçon jardinier car la serre ne peut pas rester sans surveillance.

Figure 15

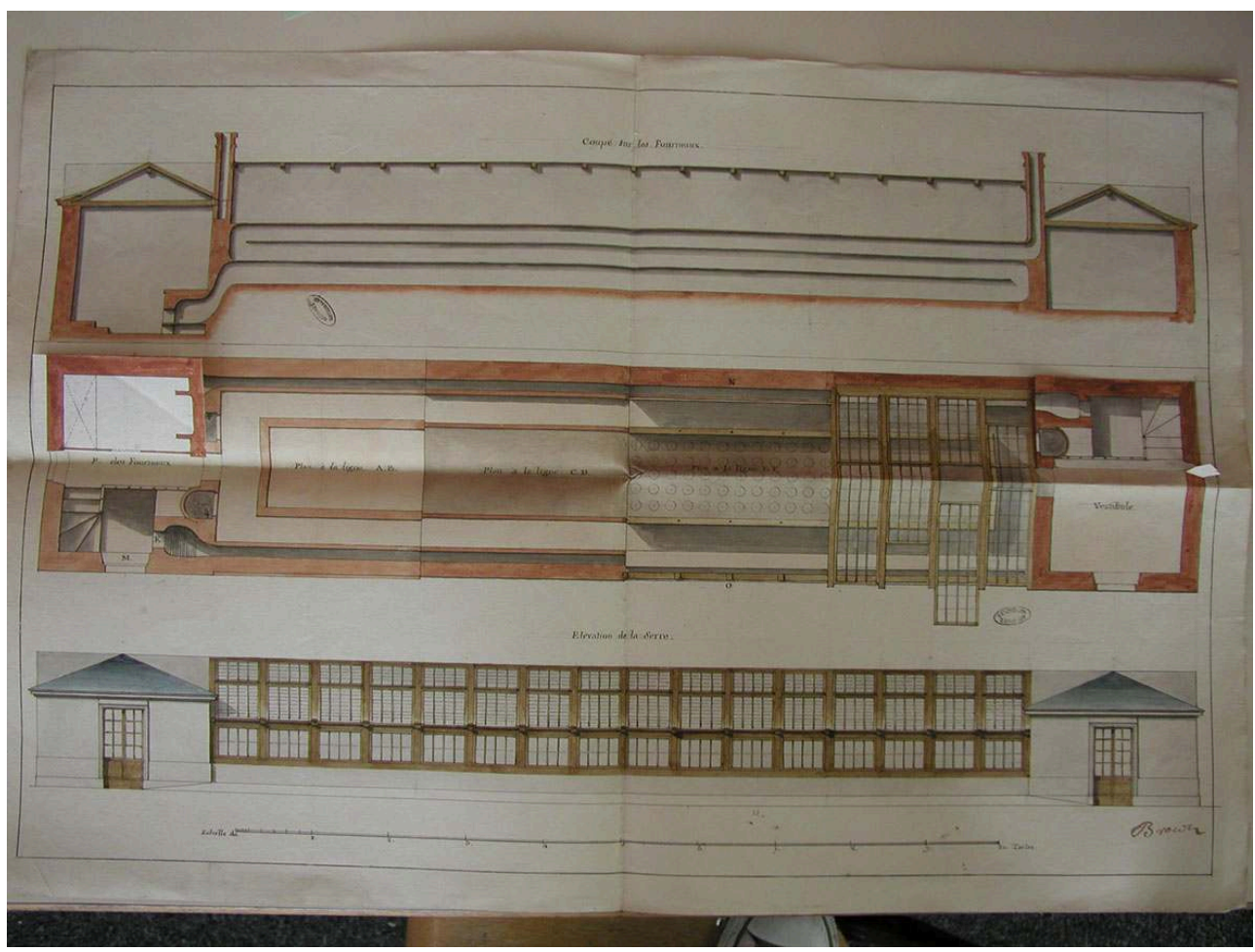

Plan en coupe de la serre à ananas (Choisy-le-Roi). AN, 01 1347-387.

Phot. Marion Rouet. (c) Marion Rouet.

Brown estime la dépense de cette grande serre à 9000 livres et il accepte de se charger de la construction pour cette somme tout en utilisant de bons matériaux dont de la brique de Bourgogne et des bois sains sans nœuds. Cependant, le montant des dépenses réalisé en 1766 s'avère bien supérieur car cette serre est une entreprise de grande envergure. Sa construction et celle de ses dépendances reviennent à 59185 livres. En effet, la maçonnerie, comprenant les terrasses pour la grande serre, les deux hangars adossés, les deux pavillons aux extrémités de la serre et les murs de clôture pour le jardin des serres, s'élève à 24497 livres. La fourniture des stores de toile forte cirée des deux côtés et garnis de plaques de tôle et fer blanc pour les fermetures, des châssis garnis, des tringles, des rouleaux de bois et des cordages pour les faire manœuvrer est estimée 2000 livres. Il y a également 1500 livres prévues pour du treillage.

Cette grande serre forme un nouvel ensemble dans les jardins de Choisy. Elle est complétée par dix petites serres en briques de Bourgogne, soit une dépense de 37363 livres. Le jardin des serres comprend quatre bâches, quatre puisards et quatre regards ainsi que quatre bancs de pierre et 270 toises de bordures en bois de chêne peintes en vert, soit une dépense supplémentaire de 5567 livres. Enfin, la construction du logement du jardinier est estimée à 24403 livres tandis que celle de la fruiterie est 
évaluée à plus de 17000 livres. Louis XV ne recule devant aucun sacrifice pour développer la culture des ananas en France. Si la serre permet d'accueillir 430 pieds d'ananas sur couche, pas moins de 3000 plantes doivent être réparties dans dix couches devant celle-ci.

En 1765 également, Claude Richard, jardinier de Trianon, a mis au point une nouvelle serre pour les ananas en tenant compte du rayonnement du soleil pour bénéficier du maximum d'ensoleillement ${ }^{46}$ (fig. 16). De plus, l'implantation de cette serre chauffée est faite pour que l'ombre portée au jardin ne soit pas trop importante. Tandis que les plantes en pots reposent dans la tannée qui s'appuie sur le sol naturel, la structure de la serre est maçonnée. Le mur de soutien, de 9 pieds 6 pouces de haut ${ }^{47}$, est conçu pour recevoir deux cheminées et diffuser au mieux la chaleur par l'intermédiaire de briques. De plus, au plus près des cultures d'ananas, le mur de soutien opposé est fait de deux assisses et mesure 2 pieds 2 pouces de haut ${ }^{48}$. Il contient une petite cheminée de 10 pouces de large, couverte de carreaux, ainsi qu'un espace vide couvert de briques pour emmagasiner la chaleur et éviter de brûler la tannée. Une dalle de pierre est prévue pour permettre la promenade sur un trottoir de deux pieds de large.

Figure 16

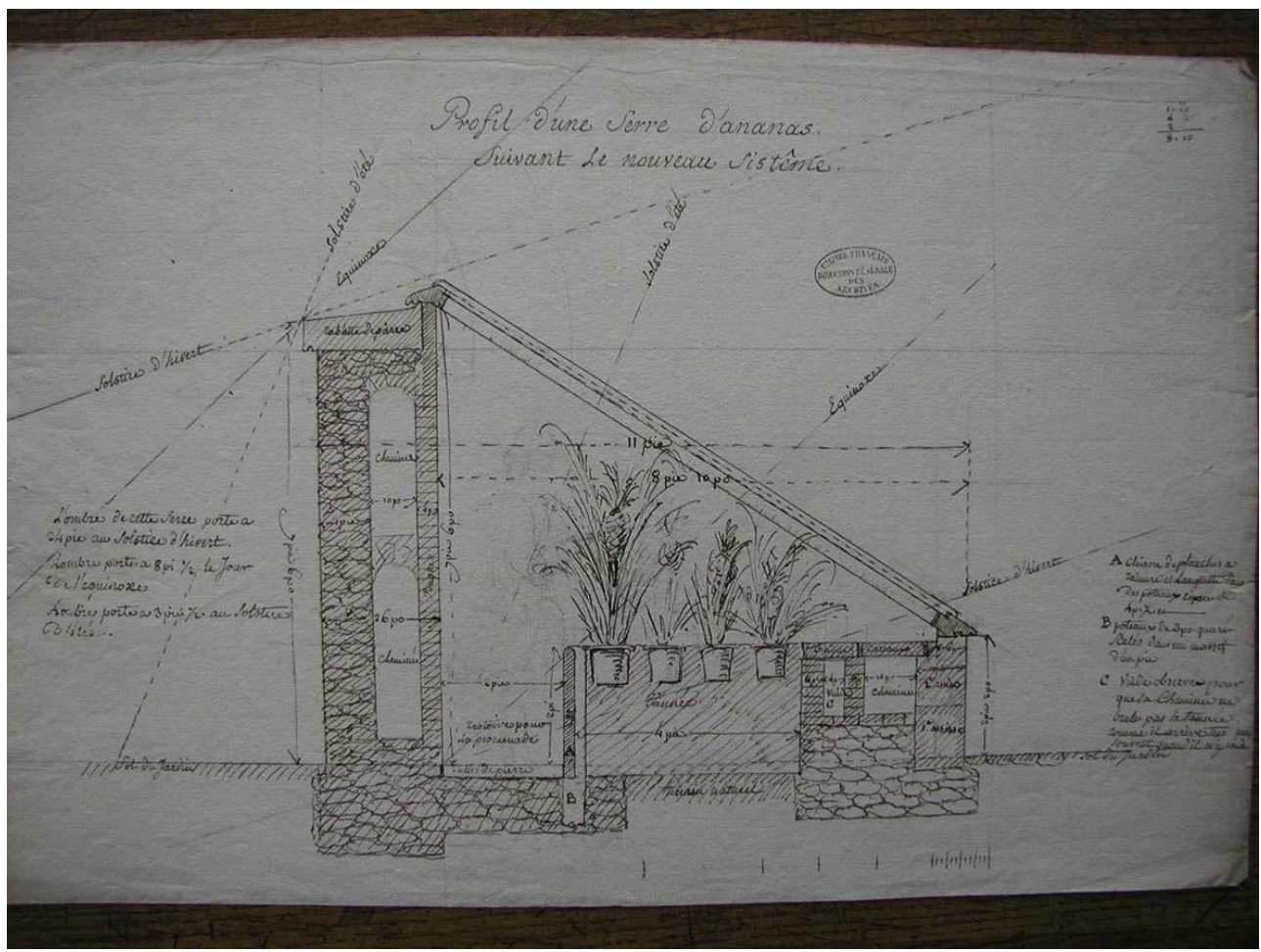

"Profil pour une serre d'ananas suivant le sisteme du sieur Richard», (Trianon, 1765). AN, O' 1887-1 $n^{\circ} 82$.

Phot. Marion Rouet. (c) Marion Rouet.

Toutefois, l'administration des Bâtiments du roi recherche un moyen de restreindre le coût de ces cultures, notamment les frais d'exploitation. Ainsi, Jaubert, qui a appris la maîtrise de la culture des ananas à Brunoy par le jardinier Martin, pense pouvoir réduire les fournitures en bois de chauffage en 1774. Brown, jardinier de Choisy, consomme en moyenne par an 41 cordes de bois et 700 fagots, ce qui revient à 1832 livres en comptant le sciage et les chandelles nécessaires. En comparaison, le 
jardinier de Brunoy brûle chaque année 8 cordes de bois et 100 petits fagots pour la même surface de serres, soit 66 toises $^{49}$. Cette différence notoire s'explique par la mauvaise qualité du bois fourni à Choisy mais aussi, d'après le mémoire, par les abus commis par Brown. Tout cela a été rédigé dans le but d'évincer Brown du potager de Choisy. Les réductions proposées paraissent forcées aux yeux de la direction des Bâtiments. Ce mémoire reste donc sans conséquence sur la gestion des serres à ananas et du potager de Choisy. Nous pouvons souligner une nouvelle fois la rancœur que témoigne le contrôleur du département de Choisy à l'encontre de ce jardinier manifestement trop indépendant! Pourtant, en 1790, il est précisé que les ananas "paroissent d'ailleurs dans un deperissement tres notable et qu'on ne devoit pas prevoir de la gestion ou culture confiée au sieur Brown ${ }^{50}$. En effet, le jardinier a laissé se dégrader le matériel (toiles cirées, paillassons). L'administration semble désarmée car il est précisé que le jardinier n'a pas de supérieur !

Pour se prémunir de la mauvaise odeur de renfermé qui domine dans les serres, Parant de Martigné rapporte un procédé qu'il a vu appliqué chez un grand cultivateur anglais membre de la Société d'agriculture de Londres. Dans la propriété de ce dernier, à 80 miles de Londres,

57 « il y a une chose de son invantion tres utiles pour les serres chaudes laquelle il n'a pas encore rendu publique parce qu'il veut être le seul à l'avoir. C'est un moyen de degresser l'air et otter l'odeur de renfermé qui est dans les serres chaudes, de sorte qu'on se promenes avec plaisir dans la siene et la pointe des feuilles des ananas ni celles des arbres ne sont pas jaunes ${ }^{51}$.»

Parant de Martigné est allé en Angleterre dans le but de voir et d'apprendre la manière de faire, dans ce pays, dans les domaines de l'agriculture, des arts et des métiers. Il fait part de cette découverte au roi de France pour lui faire plaisir car on lui a dit que le roi aimait ses serres chaudes. D'après lui, il n'y a pas d'aménagement spécifique à faire et le procédé peut être mis en place en une demi-journée. Nous constatons une nouvelle fois qu'il n'est nullement question de goût mais que l'administration royale ne se soucie que de l'esthétique des productions. La culture de l'ananas s'avère être une course technologique et politique, affirmant la domination de la nature ainsi que la supériorité de la France.

Les serres chaudes sont très à la mode en Europe du Nord dans la seconde moitié du XVIII ${ }^{e}$ siècle. Georges-Louis Le Rouge a gravé en 1775 les serres chaudes de la princesse de Galles à Kew (Royaume-Uni) ainsi que sur la même planche (fig. 17), les serres chaudes d'ananas à Utrecht (Pays-Bas). Ces dernières sont différentes de celles proposées par les jardiniers du roi de France. En effet, elles sont construites en bois tandis que les plants d'ananas reposent sur une couche de fumier recouverte d'une couche de sciure de bois. Cette planche comprend également des plans et des détails de serres à pêchers et de serres pour faire mûrir le raisin. Ces serres chauffées, qui produisent des fruits contre-nature, vont devenir le symbole du luxe déployé par une société jugée décadente ou tombée en démence par certains à la fin de l'Ancien Régime. Cet aspect se retrouve également dans la production des légumes. 


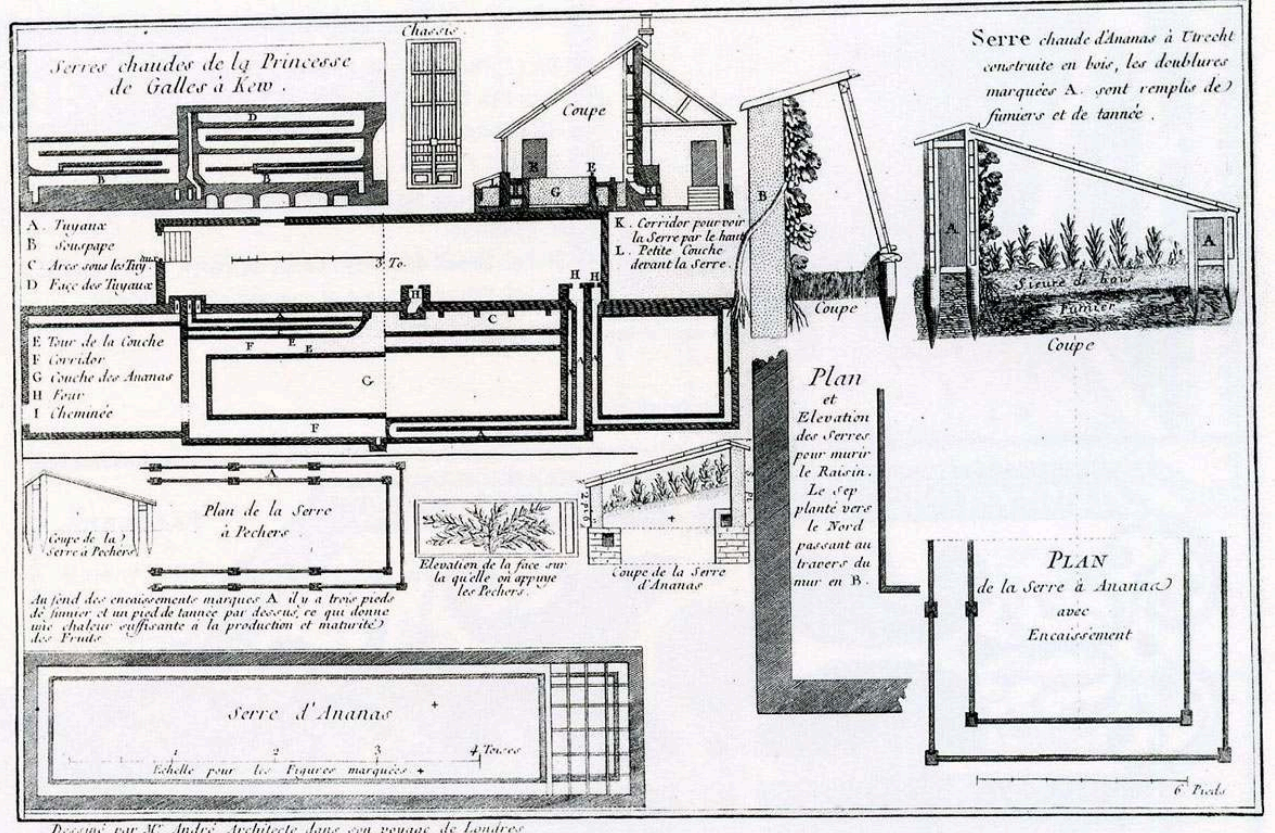

Georges-Louis Le Rouge, « Serres chaudes de la princesse de Galles à Kew et serre chaude d'ananas à Utrecht ». Jardins anglo-chinois à la mode ou Détail des nouveaux jardins à la mode. Paris : 1775. BnF, Cabinet des estampes, Hd-89 (2)-Pet. fol.

(c) BnF.

60 La culture forcée devient en effet à la fin du XVIII ${ }^{\mathrm{e}}$ siècle le symbole de la décadence de la noblesse française, qui consomme à grands frais des produits provenant du fumier. Contrairement à la pratique salutaire du jardinage vantée quelques années plus tôt, les primeurs caractérisent la perte de repères et de valeurs de cette société. L'abbé Rozier ne justifie l'existence de ces cultures que par le désir de luxe et le profit des maraîchers. Toutefois, il assure que les nobles autour de Paris ne sont plus en mesure de savoir ce qui est bon et de ce que signifie la nature. Il est vrai qu'il n'est plus question de saveur ou de goût pour définir un bon fruit: c'est la précocité de sa maturité qui détermine toute sa valeur.

La richesse enfante le luxe, \& le luxe multiplie les besoins, sur-tout les besoins superflus. Le financier veut à prix d'argent soumettre la nature à ses goûts; rapprocher, pour ainsi dire, les climats, afin d'obtenir leurs productions diverses ; \& aidé par l'art, jouir des présens de Pomone au milieu des rigueurs de l'hiver. Ces jouissances à contre-temps flattent la vue \& la vanité ; le goût l'est-il ? C'est ce dont on se soucie bien peu ${ }^{52}$.

61 Cette vision très critique de la société est partagée par Louis-Sébastien Mercier, qui rêve d'un avenir meilleur dans L'An deux mille quatre cent quarante. Rêve s'il en fut jamais :

Tous les mets dont je goûtais n'avaient presque point d'assaisonnement et je n'en fus pas faché : je leur reconnues une saveur, un sel qui était celui que leur donne la nature et qui me parut délicieux. Je ne trouvai point de ces aliments raffinés qui ont passé par les mains de plusieurs teinturiers, de ces ragoûts, de ces jus, de ces coulis, de ces sucs échauffants qui, raréfiés dans de petits plats fort coûteux, hâtaient la destruction de l'espèce animale en même temps qu'ils brûlaient les entrailles humaines. Ce peuple n'était pas un peuple carnassier qui se ruinait pour la table et dévorait plus que la magnificience de la nature ne pouvait produire avec toutes ses facultés génératives. Si tout luxe était odieux, celui de la table paraissait un crime 
révoltant car si un riche, abusant de son opulence, gaspille les biens nourriciers de la terre, il faut nécessairement que le pauvre les achète chèrement et, de plus, se retranche d'un repas.

Les légumes, les fruits étaient tous de la saison, et l'on avait perdu le secret de faire croître dans le cœur de l'hiver des cerises détestables. On n'était pas jaloux des primeurs, on laissait faire la nature : le palais en était plus flatté et l'estomac s'en trouvait mieux. On servit au dessert des fruits excellents et l'on but d'un vin vieux $[\ldots]^{53}$.

\section{Lexique}

Arbre de haute-tige : c'est la forme la plus grande pour les arbres fruitiers de plein vent, que l'on retrouve traditionnellement dans les vergers.

Arbre de plein-vent : Arbre fruitier planté loin des murs ou des clôtures.

Arbre demi-tige : il a la même forme que le haute-tige mais il est plus petit : un tronc de 1 à $1,50 \mathrm{~m}$ pour une hauteur totale de 4 à 6 mètres.

Arbre nain : arbre fruitier qui est taillé de façon à occuper le moins d'espace possible.

Arpent : c'est une ancienne unité de mesure de surface agraire française.

Bâche : c'est le nom donné aux structures spécifiques qui accueillent les cultures d'ananas.

Bon du roi : autorisation accordée par le roi à une demande particulière.

Couche : c'est la préparation du terrain avant semis ou plantation.

La couche chaude est une technique permettant d'obtenir des cultures potagères précoces, grâce à la chaleur résultant de la fermentation de fumier frais.

Espalier : Disposition d'arbres fruitiers plantés le long d'un mur sur lequel on palisse les branches, pour favoriser l'ensoleillement et protéger des intempéries les fleurs et les fruits.

Jardinier fleuriste : il est, d'après Louis Liger, spécialisé dans « la culture universelle des fleurs, arbres, arbustes, arbrisseaux servant à l'embellissement des jardins » (LIGER, Louis. Le Jardinier fleuriste. Paris : Damien Beugnié, 1704).

Palisser : Fixer les branches et les rameaux d'un arbre fruitier, d'un arbrisseau, contre un mur à l'aide d'un treillage ou de tout autre support.

Pied : c'est une ancienne unité de mesure, que l'on estime aujourd'hui à 30,48 cm.

Planche : zone de culture que le jardinier devra préparer avant toute plantation.

Tannée : produite à partir de l'écorce de chêne, elle « sert à faire ce qu'on appelle des mottes à brûler. Les jardiniers l'emploient aussi à faire des couches chaudes. Elle est très propre pour les ananas qui ne peuvent supporter la vapeur du fumier de cheval.» (Dictionnaire universel françois et latin, vulgairement appelé Dictionnaire de Trévoux). Paris : La Compagnie des libraires associés, vol. 7, 1771, p. 974) 


\section{BIBLIOGRAPHIE}

BECCALETTO, Jacques. Encyclopédie des formes fruitières. Arles/Versailles : Actes Sud/École nationale supérieure du paysage, 2001.

BÉNETIÈRE, Marie-Hélène, CHATENET, Monique, MOSSER, Monique. Jardin. Vocabulaire typologique et technique. Paris : Éditions du patrimoine, 2000.

BOURDE, André-Jean. Agronomie et agronomes en France au XVIII ${ }^{e}$ siècle. Paris : SEVPEN, 1967.

COMBLES, De. L'École du jardin potager. Paris : Savoye, 1794 (4 éd.).

COMBLES, De. Traité de la culture des pêchers. Paris : Antoine Boudet, 1745.

DÉZALLIER D’ARGENVILLE, Antoine-Joseph. La Théorie et la pratique du jardinage. Paris : Jean Mariette, 1732 ( $3^{\mathrm{e}}$ éd.).

DÉZALLIER D'ARGENVILLE, Antoine-Nicolas. Voyage pittoresque des environs de Paris. Paris : De Bure l'aîné, 1755.

DUHAMEL DU MONCEAU, Henri-Louis. Traité des arbres fruitiers. Paris : Saillant et Desaint, 1768.

FLANDRIN, Jean-Louis. « Les fruits et légumes dans l'alimentation des élites sociales, du XIV au XVIII ${ }^{\mathrm{e}}$ siècle ». Dans MEILLER, Daniel, VANNIER, Paul. Le Grand Livre des fruits et légumes. Histoire, culture et usage. Besançon : la Manufacture, 1991, p. 156-160.

GANAY, Ernest de. Les Jardins de France et leur décor. Paris : Larousse, 1949.

GIBAULT, Georges. « Étude sur la bibliographie et la littérature horticoles anciennes ». Journal de la Société nationale d'horticulture de France, novembre 1905, p. 710-746.

GIBAULT, Georges. " Les erreurs et les préjugés dans l'ancienne horticulture ». Journal de la Société nationale d'horticulture de France, mars 1897, p. 254-277.

GIBAULT, Georges. « Les origines de la culture forcée ». Journal de la Société nationale d'horticulture de France, 1898, p. 1109-1117.

GIBAULT, Georges. Histoire des légumes. Paris : Librairie Horticole, 1912.

LA QUINTINIE, Jean-Baptiste, de. Instruction pour les jardins fruitiers et potagers. Arles/Versailles : Actes Sud/ENSP, 1999 (reprod. en fac-similé de l'éd. de 1690).

LE BERRYAIS, René. Traité des jardins ou le nouveau de La Quintinye [1775]. Caen : Manoury l'aîné, 1785 , t. 2, Des plantes potagères. (consultable sur Gallica)

LE ROUGE, Georges-Louis. Jardins à la mode et Jardins anglo-chinois. Détail des nouveaux jardins à la mode. Paris : D. Jacomet pour le Jardin de Flore, 1978 (fac-similé de l'édition de 1776-1789, consultable sur Gallica).

MERCIER, Louis-Sébastien. L'An deux mille quatre cent quarante. Rêve s'il en fut jamais [1770]. Édition Raymond Trousson. Bordeaux : Ducros, 1971.

MILLER, Philip. Dictionnaire des jardiniers. Paris : Guillot, 1785 (8édition).

QUELLIER, Florent. « Les fruits de la civilité françoise : l'engouement des élites du XVII ${ }^{\mathrm{e}}$ siècle pour le jardin fruitier-potager ». Polia, $n^{\circ}$ 8, automne 2007, p. 25-39.

QUELLIER, Florent. Des fruits et des hommes l'arboriculture fruitière en Île-de-France (vers 1600vers 1800). Rennes : PUR, 2003. 
QUELLIER, Florent. Histoire du jardin potager. Paris : Armand Colin, 2012.

ROUET, Marion. Les Potagers aristocratiques et royaux en Île-de-France (fin XVII ${ }^{e}$-fin XVIII ${ }^{e}$ siècle). Thèse de doctorat en histoire. Paris : université Paris-13, 2011.

ROZIER, abbé François. Cours complet d'agriculture théorique, pratique, économique et de médecine rurale et vétérinaire. Paris : Hôtel Serpente, 1781-1789, t. I-VIII.

ROZIER, abbé François. Cours complet d'agriculture théorique, pratique, économique et de médecine rurale et vétérinaire [1781]. Paris : Delalain fils, 1797-1805, t. IX et X.

ROZIER, abbé François. Cours complet d'agriculture théorique, pratique, économique et de médecine rurale et vétérinaire. Paris : Marchant, 1805, t. XI et XII.

ROZIER, abbé François. Nouvelle table des articles contenus dans les volumes de l'Académie royale des sciences de Paris depuis 1666 jusqu'en 1770. Paris : Ruault.

WHEELER, William. Le Potager du roi. Fruits et légumes du château de Versailles. Paris : Somogy Éditions d'art/Aventurine, 1998.

WHEELER, William. Les plus beaux potagers, du jardin ouvrier au potager du château. Paris : Somogy Éditions d'art, 1999.

\section{NOTES}

1. - Voir le site: https://ich.unesco.org/fr/RL/le-repas-gastronomique-des-francais-00437 [consulté le 18/11/2019].

2. - $\mathrm{AN}, \mathrm{O}^{1}$ 1347-227, « Plan pour fixer les parties de jardins qui seront confiées aux soins des sieurs Gondoin et Brown », 29 juillet 1763.

3. - QUELLIER, Florent. Histoire du jardin potager. Paris : Armand Colin, 2012, p. 93.

4. - Environ 127 ares.

5. - Vitry-sur-Seine, Val-de-Marne.

6. - AN, $\mathrm{O}^{1} 1351 / 36,1755$, le potager particulier du roi de Choisy et son environnement.

7. - Environ $2 \mathrm{~m}$.

8. $-1,3 \mathrm{~m}$.

9. - AN, $\mathrm{O}^{1} 1348 / 325$.

10. - MAYER, Johann Prokop. Pomona Franconica. Description des arbres fruitiers les plus connus et les plus estimés en Europe. Nuremberg : Adam Wolfgang Winterschmidt, 1776-1801, t. II, Cerises, neffles et azeroles, pêches, 1779, p. 25.

11. - AN, $\mathrm{O}^{1} 2112^{4}$, lettre de Mme de Case, le 12 novembre 1786. Il semble qu'il soit question de cerisier d'Angleterre.

12. - AN, $O^{1} 1348 / 161$, lettre du contrôleur des Bâtiments du roi du département de Choisy, le 5 octobre 1769.

13. - ROUSSEAU, Jean-Jacques. CEuvres. Paris : Th. Desoer, 1822-1825, Confessions [1762], 1822, t. I, p. 236.

14. - AN, $\mathrm{O}^{1} 1350 / 13$.

15. - AN, $\mathrm{O}^{1} 1343 / 6$ bis.

16. - Il est, depuis 1775, inspecteur général des jardins fruitiers et potagers royaux. Voir : La dynastie des Le Normand - un jardin historique - Le potager du roi. Voir le site : http://www.potagerdu-roi.fr/site/pot_histoire/dynastie_le_normand.htm [consulté le 18/11/2019].

17. - QUELLIER, Florent. Des fruits et des hommes. Rennes : PUR, 2003, p. 68.

18. - Voir les jardins de biais mis en place par La Quintinie dans le Potager du roi à Versailles. 
19. - Chaque année, tous les jardiniers royaux adressent à leur administration une demande de « regarni », c'est-à-dire la liste des arbres nécessaires pour remplacer les arbres malades ou trop vieux au sein du jardin dont ils ont la charge.

20. - AN, O $O^{1}$ 1347/176, Choisy, 12 mars 1763.

21. - AN, $\mathrm{O}^{1} 1431 / 369$, lettre du directeur des Bâtiments du roi, le marquis de Marigny, du 4 décembre 1755 .

22. - SCHABOL, Abbé Roger. La Pratique du jardinage [1767]. Paris : chez les frères Debure, 1774, 2 t. ; Id. La Théorie du jardinage. Paris : chez les frères Debure, 1774.

23. - $\mathrm{AN}, \mathrm{O}^{1} 1347 / 145$. Lettre à Michel-Barthélemy Hazon, contrôleur général des Bâtiments du département de Choisy, du 24 novembre 1762.

24. - Voir le site : https://mursapeches.blog/qui-sommes-nous/lhistoire-des-murs/ [consulté le 18/11/2019].

25. - AN, $\mathrm{O}^{1} 1347 / 213$, lettre du contrôleur du département de Choisy, 14 juin 1763.

26. - AN, $\mathrm{O}^{1} 1347 / 311$, lettre du contrôleur du département de Choisy, 3 août 1764.

27. - AN, $O^{1} 1347 / 333$, Direction générale, porte la mention « bon ».

28. - COMBLES, De. Traité de la culture des pêchers [1745]. Paris: Delaguette et Le Prieur, 1750, p. 152.

29. - Mercier Louis-Sébastien, Tableau de Paris, Amsterdam, 1782, tome II, p. 307.

30. - Simon-Charles Boutin, fils d'un receveur des finances, crée en 1766 rue Saint-Lazare à Paris un jardin qui devient rapidement un lieu de divertissement réputé.

31. - Louis Philippe Joseph D’Orléans fit réaliser le jardin de Monceau par Louis de Carmontelle, qui est achevé en 1778, tandis que Louis-Antoine de Gontaut-Biron, maréchal de Biron, achète en 1753 un hôtel rue de Varenne dont une partie des jardins est réaménagée à l'anglaise.

32. - Voir glossaire ci-dessous.

33. - AN, $\mathrm{O}^{1}$ 1347/500, projet d'entretien pour le sieur Brown à Choisy, juin 1766.

34. - AN, $\mathrm{O}^{1} 1846-5 \mathrm{n}^{\circ} 22$, Plan, coupe et élévation d'une serre chaude, s.d..

35. - Environ $3,2 \mathrm{~m}$.

36. - AN, $\mathrm{O}^{1} 1352 / 87$.

37. - Une banque d'Amsterdam, également active dans le commerce du tabac d'Amérique, porte ce nom.

38. - AN, $\mathrm{O}^{1} 1343 / 105$.

39. - $\mathrm{AN}, \mathrm{O}^{1} 1347 / 297$.

40. - Charles Fouquet, duc de Belle-Isle (1684-1761).

41. - AN, $\mathrm{O}^{1} 1347 / 226$.

42. - AN, $\mathrm{O}^{1} 1347 / 400,1^{\text {er }}$ septembre 1765.

43. - AN, $\mathrm{O}^{1} 1347 / 510$ et $\mathrm{O}^{1} 1347 / 513$.

44. - AN, $\mathrm{O}^{1} 1347 / 388,28$ juin 1765 .

45. - AN, $\mathrm{O}^{1} 1347 / 387$.

46. - AN, $\mathrm{O}^{1} 1887-1 \mathrm{n}^{\circ} 82$, « Profil pour une serre d'ananas suivant le sisteme du sieur Richard pour être placée en terrasse derrière la petite orangerie, qui n'a plus lieu en $1765 »$.

47. - Soit environ $3 \mathrm{~m}$.

48. $-70 \mathrm{~cm}$.

49. - Environ $130 \mathrm{~m}$

50. - AN, $\mathrm{O}^{1}$ 1350/273, observation faite le 2 octobre 1790. 
51. - AN, $\mathrm{O}^{1} 2106^{2}$, lettre du 8 août 1765.

52. - ROZIER, abbé François. Cours complet d'agriculture [1781]. Paris : Hôtel Serpente, 1785, t. VI, p. 2.

53. - MERCIER, Louis-Sébastien. L'An deux mille quatre cent quarante. Rêve s'il en fut jamais [1770]. Édition Raymond Trousson ; Bordeaux : Ducros, 1971 chap. XLV, « L'avantsouper », p. 385-386.

\section{RÉSUMÉS}

Quel est le goût du roi de France au cours de la seconde moitié du XviII siècle en matière de fruits? L'administration des Bâtiments du roi accorde une grande importance au travers de sa correspondance avec ses jardiniers aux cultures des cerises, pêches et ananas. Grâce notamment à l'exemple du domaine royal de Choisy-le-Roi, nous nous questionnerons sur la façon dont les arbres fruitiers étaient cultivés afin de satisfaire Louis XV et si le goût, seul, était recherché ou bien si d'autres critères étaient pris en compte pour apprécier un fruit à la table du roi. L'esthétique et surtout la précocité confèrent à certaines de ces productions un caractère exceptionnel. La rareté triomphe du goût grâce aux perfectionnements des techniques de production par l'utilisation de serres puis d'abri chauffé. Cela révolutionne les conditions de culture au sein des potagers royaux.

Where fruit was concerned, what did the King like during the second half of the eighteenth century? According to its correspondence with its gardeners, the administration of the royal buildings paid particular attention to the cultivation of cherry trees, peach trees and pineapple plants. Looking at the royal estates of Choisy-le-Roi, we will try to understand how fruit trees were cultivated in order to satisfy Louis XV's tastes and if indeed taste was the only criterion involved, or if other considerations were not also taken into account in bringing fruit to the royal table. Aesthetic considerations and precocity in the season could also give certain fruits exceptional favour. Rarity was perhaps more important than flavour, thanks to progress in production techniques, such as greenhouses and hot houses. These brought a revolution to the conditions of tree and plant culture in the royal gardens.

\section{INDEX}

Keywords : cherry, peach, pineapple, greenhouse, vegetable garden, taste, Choisy-le-Roi, espalier, Louis XV, technique, season, savoir-faire, fruit tree

Mots-clés : cerise, pêche, ananas, serre, jardin potager, goût, Choisy-le-Roi, espalier, Louis XV, technique, saison, savoir-faire, arbre fruitier

\section{AUTEUR}

\section{MARION ROUET}

Enseignante Marion.Rouet@ac-versailles.fr 